\title{
Theory Of Enhancement
}

\author{
Formation and Expansion of the Universe
}

\author{
Trevor Mohlala
}

DOI: 10.29322/IJSRP.11.10.2021.p11876

http://dx.doi.org/10.29322/IJSRP.11.10.2021.p11876

\begin{abstract}
The environment originates from mass, $(\boldsymbol{m}=\mathbf{1})$, and at the point of origin mass is governed by the subliminal law of motion, and the root force of mass that accounts for its potential energy. When mass breaks into motion, it is propelled by force of gravity and mass in motion is dark matter. Mass moves because it goes into a process of decay. This movement of mass is considered the primary motion of dark matter, it fulfils the first law of motion and engage the second law and the supportive law of motion activates and provide support, thereby giving birth to anti-dark matter. Anti-dark matter is the secondary motion of mass activated to support the primary motion of mass. Dark matter has three waves and anti-dark matter has two waves. When the third wave of dark matter collides at a very high gravitational speed, it forms the first nucleus, bringing forth the first matter, whilst on the opposite, the second wave of anti-dark matter collides to form antimatter. This is the point where the enhancement of particles and antiparticles begins. The enhancement of particles is only for a single purpose, to balance the third wave of dark matter, so that particles can thrive in an environment of their own. On the other hand, antiparticles enhance to balance particles, and thereby balancing the second wave of anti-dark matter. When dark matter and anti-dark matter is balanced by the particles and antiparticles within them, this results in the balance mass. This total balance means, the potential energy of mass is now fulfilled.
\end{abstract}

Index Terms- Theory of Enhancement, Universal Balance, Universal Expansion Universe formation.

\section{INTRODUCTION}

The nucleus of matter is the innermost realm of the environment, followed by the nucleus of antimatter, and then the third wave of dark matter followed by the second wave of anti-dark matter, and then the second wave of dark matter followed by the first wave of dark matter with mass being the outermost realm of the environment. Dark matter and anti-dark matter are mass moving at the speed of gravity, whilst particles and antiparticles are hard at work to balance dark matter and anti-dark matter respectively. The entire physical environment is transformed by motion, and this requires energy. The energy regulating the motion and balance of the environment comes from mass, and there, it is stored as potential energy. Of all the known laws of motion, there is a law of motion that is concerned with potential motion, and this is the subliminal law of motion, the other law of motion being introduced is concerned with supportive motions, and this is the supportive law of motion. Dark matter collides in the process of the big bag and form the known particles of the universe, anti-dark matter collides to balance the activity of dark matter collision and thereby form the antiparticles of the universe. When the second wave of anti-dark matter collides, it forms the first antiparticles (antineutron and antiproton). Antiparticles are here to balance particles, and they continue following the same laws governing motion in the entire environment, meaning the same laws and forces continue but only opposite the motion and forces of particles. The movement of an antiproton occurs opposite that of a proton and therefore an antiproton becomes negative. The force holding together the antineutron and antiproton is therefore the strong nuclear force. The movement of a positron occurring opposite an electron, earns the positron a positive charge and the force regulating the positron environment is the weak nuclear force. The final force results from the electromagnetic interaction of the antiproton and the positron and it is the electromagnetic force of antiparticles. All these antimatter forces work together to balance the force of gravity that is exerted on the antiparticles by anti-dark matter. The latter scenario is the same with particles as already known, as the strong nuclear force, weak nuclear force and the electromagnetic forces of particles work together to balance the force of gravity exerted to the articles by dark matter. When particles enhance, dark matter enhances thereby forming wormholes in the centre of balanced blackholes. The same happens with the enhancement of antiparticles as it results in the enhancement of anti-wormholes in the centre of opposite blackholes. These dynamic motions of the environment results in the multidimensional nature of the environment, with every dimension in motion, fulfilling its purpose in balancing the environment. As the enhancement of particles and antiparticles progresses, they grow in energy until a point where balance is reached:

1. The root force holds together motionless mass and is governed by the subliminal law of motion.

2. Dark matter is first balanced by anti-dark matter leading to the formation of particles which are balanced by antiparticles.

3. The supportive law of motion facilitates the fulfilment of the second law of motion, without the supportive law of motion, Newton's second law of motion would govern perpetual motion and Newton's third law of motion would be impossible.

4. The nuclear enhancement table presents the theoretical expansion of the universe based on the quantum realm of particles and antiparticles. 
5. Completely transformed particles and antiparticles of mass have no need to move as they have fulfilled all the laws of motion with their accompanying forces, they have reached a fulfilled state of mass and are now held in position by the root force of mass.

\section{MASS}

Mass is the basic foundation of the entire universe, and the universe is its expression. It is a realm where all motions, forces, and balance are conspired. Even though the law governing this realm may be elusive, but it is a law that governs motion before motion is realised. The subliminal law of motion is a law that accounts for every other motion before that motion occurs and it is accounted for as potential energy which is governed by the root force of the environment. In everyday life, one may think of mass in terms of the measurement of its heaviness. However, Kilogram, grams, milligrams and so forth are the measurements used to determine how much heavy mass is.

\section{The subliminal law of motion}

The intuitive level of a particle is governed by the root force of mass that produces the sum of all observable motions through the other physical forces of the environment and the root force is expressed as potential energy.

$$
E_{p}=E_{\text {universal system }}+E_{\text {dark }}
$$

The root force carries the potential motion of a particle. The root force of a particle is therefore the sum of all the potential physical forces of particles and antiparticles. This is the law that drives natural selection and eventually natural evolution. Therefore, the potential energy is the sum of all the expressed energy of the particle.

The total energy is considered from the waves of dark matter and anti-dark matter, the unified field of the matter and the unified field of antimatter. This is the sum of the energy that mass has at the potential level, and it uses this energy to work and balance itself effectively.

Dark energy:

$E_{\text {dark }}=E_{\text {dark matter }}+E_{\text {antidark matter }}$

$E_{\text {dark }}=(1+2+4)+(1+2)=10$

There are five waves of dark matter and anti-dark matter that collectively results to a dark energy of ten.

The energy of the universal system $\boldsymbol{E}_{\text {universal system }}=\boldsymbol{E}_{\text {matter }}+\boldsymbol{E}_{\text {antimatter }}$

Then, $\quad E_{\text {universal system }}=\mathbf{4} \boldsymbol{N}_{\mathbf{1}}+\mathbf{4} \boldsymbol{N}_{2}$ (the nucleus of matter is denoted 1 and that of antimatter is 2 )

$$
\begin{aligned}
& E_{\text {universal system }}=4\left(4 r^{2}+4 r\right)+4\left(2 r^{2}+2 r\right) \\
\therefore \quad & E_{\text {universal system }}=24 r^{2}+24 r
\end{aligned}
$$

The total potential energy:

Then, $\quad E_{p}=E_{\text {universal system }}+\boldsymbol{E}_{\text {dark }}$

$\therefore \quad E_{p}=24 r^{2}+24 r+10$

This is the potential energy concealed within mass before the formation of dark matter and the universal system.

Mass is only (1) and it does not change, however, the speed at which, mass is moving changes and as the speed increases, the heaviness of mass decreases. At the level of dark matter and anti-dark matter, the speed of mass is measured as gravity, depending on the wave of dark matter or anti-dark matter respectively. At the level of particles and antiparticles, the speed of mass is measured as velocity and there are many different levels of particles and antiparticles where mass is in motion, with light particles being the fastest. This means, the most liberated nature of mass is a light particle.

However, the root force of mass cannot be accounted for traditionally through movement and speed as its activities are mass energised by potential energy. Therefore, the root force of mass is already accounted for above as the total potential energy of mass, and therefore mass does not move from point $\mathrm{A}$ to point $\mathrm{B}$, but dark matter, anti-dark matter, particles and antiparticles move at a certain speed, and as such, it all happens within mass at rest.

\section{The root force of mass}

This publication is licensed under Creative Commons Attribution CC BY. 


\section{The force responsible for the balance of motionless mass.}

Motionless mass is found before the first wave of dark matter, where mass has a need to move and fulfil itself, and gain motionless mass if found where mass has completely transformed itself into light, where mass has totally fulfilled itself.

\section{DARK MATTER AND ANTI-MATTER}

Fig1: Summary of the environment

Fig1a

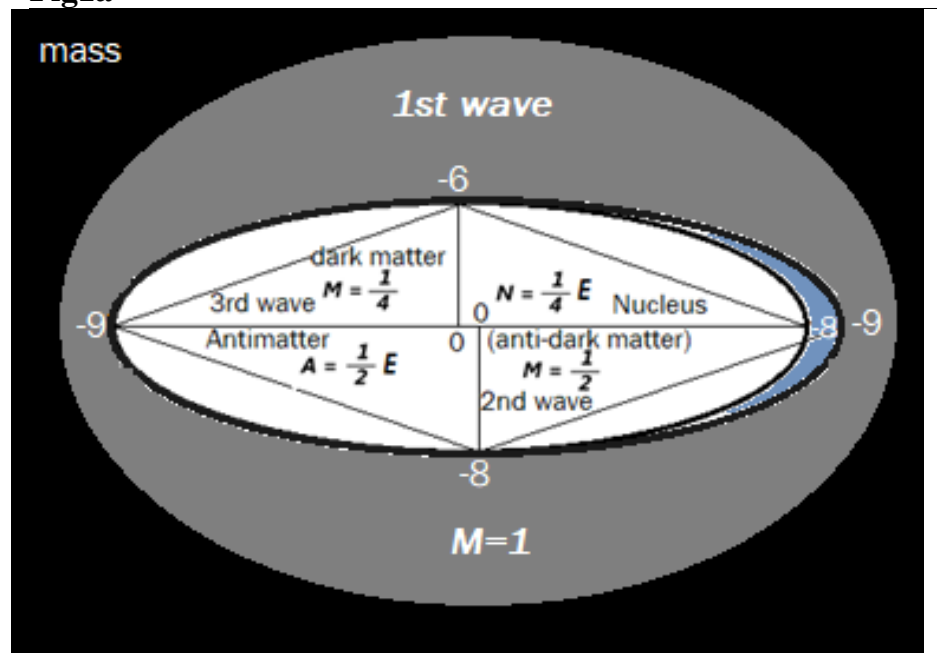

Fig1b

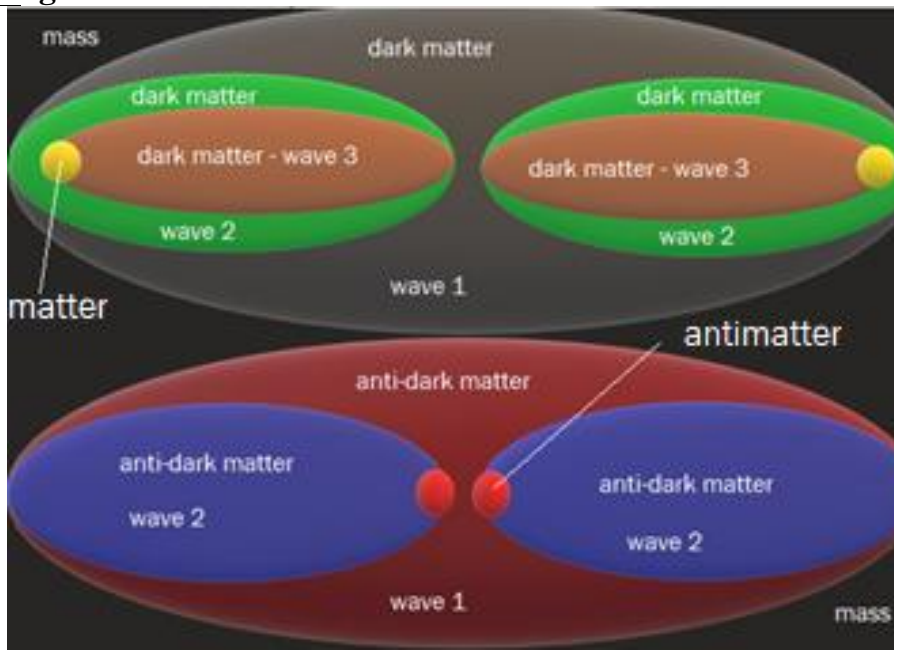

Note: When analysing figla, you are moving from the outermost realm, being mass at rest to the deepest realm of particles.

Fig1a

The figure shows the movement of dark matter from the first wave at $(-9)$ to the third wave of dark matter at $(-6)$ and then the formation of the nucleus particles, this forms the upper triangle in fig1a. The lower triangle shows the movement of anti-dark matter from $(-9)$ to the second wave of anti-dark matter at $(-8)$ and then the formation of the nucleus antiparticles. If the particles and antiparticles formed were superior in force to the waves of energy that formed them, there would be no need for further expansion of the universe. However, that is not the case and therefore the forces of matter and antimatter align elegantly for the enhancement of their own particles and antiparticles.

Fig1b

This figure shows the opposite nature of dark matter versus anti-dark matter, and particles versus antiparticles. A closer observation of the position of particles versus antiparticles provides a logical idea why their movements oppose. The figure also shows that completely transformed mass is at the innermost level of the environment.

Matter $=\frac{\text { mass }}{\text { force }}$

Then $\boldsymbol{M}=\frac{\boldsymbol{m}}{\boldsymbol{m a}}$

Therefore: $\boldsymbol{M}=\frac{\boldsymbol{m}}{\boldsymbol{m} \boldsymbol{g}}$ (because gravity is acceleration)

Thus $\boldsymbol{M}=\frac{\mathbf{1}}{\boldsymbol{g}}$

Mass is divided by a force that gets it into motion, and before the formation of the nucleus, the force that divides and move mass is the force of gravity as displayed above. This is the stage where mass becomes dark matter because it is now in motion, and it is propelled by the force of gravity. Dark matter finds support from its opposing counterpart, anti-dark matter which is also propelled by the force of gravity. Gravity propels mass until the nucleus is formed, where different forces propel mass and the forces are: strong nuclear force, weak nuclear force and the electromagnetic force, at this stage mass becomes matter and antimatter. 


\section{Dark matter - Primary motion}

At wave 1: $\boldsymbol{M}=\frac{\mathbf{1}}{\mathbf{1}}=\mathbf{1}\left(1^{\text {st }}\right.$ law of motion $)$

The force of dark matter: $\boldsymbol{f}=\boldsymbol{m g}=\mathbf{1} \times \mathbf{1}=\mathbf{1}$

At wave $2: \boldsymbol{M}=\frac{\mathbf{1}}{\mathbf{2}}\left(2^{\text {nd }}\right.$ law of motion $)$

The force of dark matter: $\boldsymbol{f}=\boldsymbol{m} \boldsymbol{g}=\mathbf{1} \times \mathbf{2}=\mathbf{2}$

At wave 3: $\boldsymbol{M}=\frac{\mathbf{1}}{\mathbf{2}} \times \frac{\mathbf{1}}{\mathbf{2}}=\frac{\mathbf{1}}{\mathbf{4}}\left(3^{\text {rd }}\right.$ law of motion $)$

Let's find gravity at wave 3:

The force of dark matter: $\boldsymbol{f}=\boldsymbol{m g}=\mathbf{1} \times \mathbf{4}=\mathbf{4}$

\section{Anti-dark matter - Secondary motion}

Opposite the $2^{\text {nd }}$ wave of dark matter, the supportive law of motion activates as it needs to balance the motion of the second wave of dark matter.

Opposite the $2^{\text {nd }}$ wave of the primary motion, the $1^{\text {st }}$ wave of the secondary motion activates: $\boldsymbol{M}=\frac{\mathbf{1}}{\mathbf{1}}=\mathbf{1}$ (Supportive law)

The force of anti-dark matter: $\boldsymbol{f}=\boldsymbol{m g}=\mathbf{1} \times \mathbf{1}=\mathbf{1}$

Opposite the $3^{\text {rd }}$ wave of the primary motion, the $2^{\text {nd }}$ wave of the secondary motion activates: $\boldsymbol{M}=\frac{\mathbf{1}}{\mathbf{2}}$ ( $3^{\text {rd }}$ law of motion $)$

The force of anti-dark matter: $\boldsymbol{f}=\boldsymbol{m} \boldsymbol{g}=\mathbf{1} \times \mathbf{2}=\mathbf{2}$

Table 2. Formation of the nucleus

\section{Nuclear formation - Matter}

\section{Nuclear formation - Antimatter}

The particle $\boldsymbol{E}=\boldsymbol{m}_{\mathbf{1}} \times \boldsymbol{m}_{\mathbf{2}}$ forms from the collision of mass in motion driven by the force of gravity forming the nuclear particles. Also, in the secondary motion antimatter is formed opposite the nucleus

$$
\boldsymbol{N}=\frac{\mathbf{1}}{\mathbf{4}} \boldsymbol{E}
$$

The particle inside the nucleus forms the radius of the nucleus: $\boldsymbol{r}=\sqrt{N}$

and therefore $N=r^{2}$

also meaning that $r^{2}=\frac{1}{4} E$

and this translate to $\boldsymbol{E}_{1}=\mathbf{4}=\boldsymbol{r r}^{2}$

\section{Electron formation}

The radius of the nucleus works to balance the nucleus against the third wave of dark matter, forming a new particle protecting the nucleus, and therefore: $\boldsymbol{E}_{2}=\mathbf{4 r}$.

now, $\boldsymbol{E}=\boldsymbol{E}_{1}+\boldsymbol{E}_{2}$

and therefore $E=4 r^{2}+4 r$

$\mathbf{4} \boldsymbol{r}^{2}$ is composed by the neutron and proton.

$\mathbf{4 r}$ is composed by the electron.

4 is mass moving at the gravitational speed of 4 .

$\boldsymbol{E}_{\mathbf{1}}=\mathbf{4} \boldsymbol{r}^{\mathbf{2}}$ this is the core particle; it is the environment of the strong nuclear force. $\boldsymbol{E}_{2}=\mathbf{4 r}$ is the environment of the weak nuclear force. The electromagnetic force is found between the positively charged protons at $\boldsymbol{E}_{\mathbf{1}}$ and the negatively charged electrons at $\boldsymbol{E}_{2}$.

$$
N=\frac{1}{2} E
$$

The particle inside antimatter forms the radius of antimatter: $r=\sqrt{N}$ and therefore $\boldsymbol{N}=\boldsymbol{r}^{2}$ also meaning that $r^{2}=\frac{1}{2} E$ and this translate to $E_{1}=2 r^{2}$

\section{Positron formation}

The radius of antimatter works to balance the core of the antimatter particle against the second wave of anti-dark matter forming a new antiparticle protecting its core: $\boldsymbol{E}_{2}=\mathbf{2 r}$.

now, $\boldsymbol{E}=\boldsymbol{E}_{1}+\boldsymbol{E}_{2}$

and therefore $E=2 r^{2}+2 r$

$2 r^{2}$ is composed by the antineutron and antiproton.

$2 \boldsymbol{r}$ is composed by a positron.

2 is mass moving at the gravitational speed of 2 .

$\boldsymbol{E}_{\mathbf{1}}=\mathbf{2} \boldsymbol{r}^{2}$ this is the core particle; it is the environment of the strong nuclear force. $\boldsymbol{E}_{2}=\mathbf{2 r}$ is the environment of the weak nuclear force. The electromagnetic force is found between the negatively charged antiprotons at $\boldsymbol{E}_{\mathbf{1}}$ and the positively charged positrons at $\boldsymbol{E}_{2}$.

The formular: $\boldsymbol{E}=\mathbf{4} \boldsymbol{r}^{\mathbf{2}}+\mathbf{4 r}$, complete energy field of particles.

$\boldsymbol{E}=2 r^{2}+2 r$, complete energy field of antiparticles. 


\section{The supportive law of motion}

\section{The energy of a balanced particle is twice the energy of the particle engaged in the second law of motion.}

$\boldsymbol{E}_{\text {support }}=\mathbf{2} \boldsymbol{E}_{\text {applied }}-$ the force of the balanced particle is twice the force of the initial particle.

When $E_{2}=\frac{1}{2}$, meaning $4 r=\frac{1}{2}$, making $E=4 r^{2}+2 r$, support is provided from the intuitive level of the particle such that $4 r$ is achieved, because this condition means this particle is experiencing resistance from the gravity against it. Thus, support is provided to ensure the successful enhancement of the particle.

Fig2: Natural balance

Fig2a nuclear balance

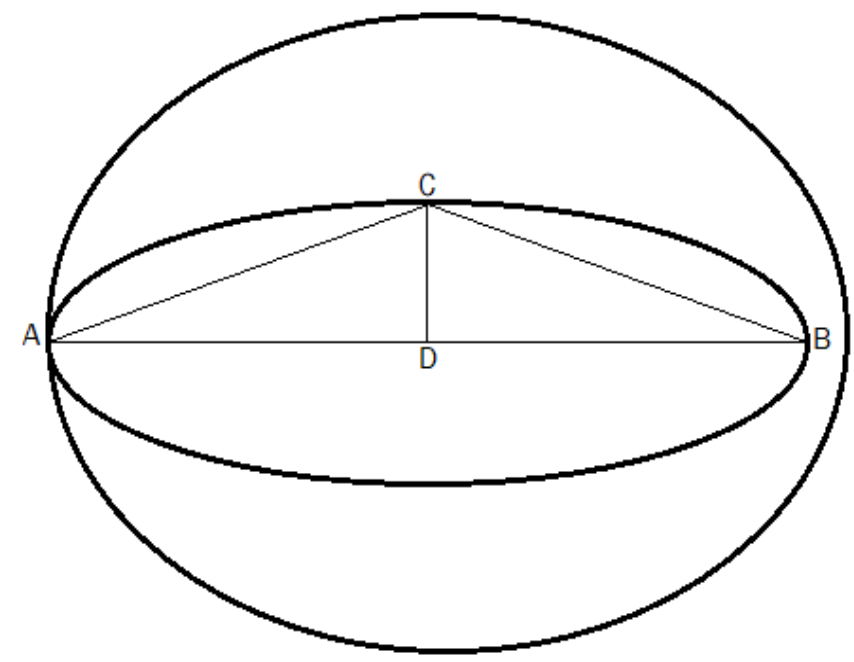

Fig2b antimatter balance

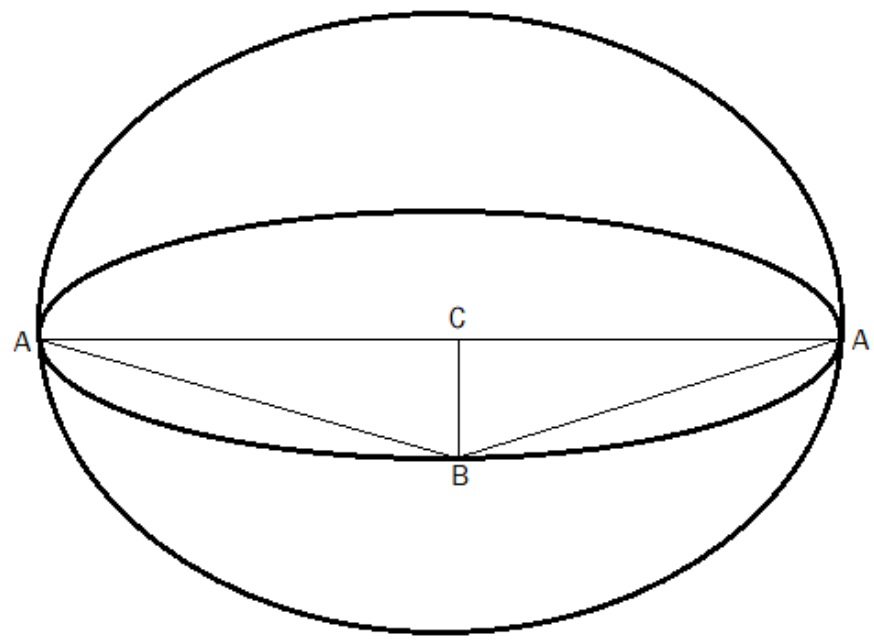

Figure 2 shows the formation of figure 1, when the primary motion begins at figure $2 \mathrm{a}$, mass moves from point A to point B. However, when the second law of motion is engaged, the particles will balance if they are of equal mass and equal speed. Hence to balance wave 2 of dark at B, two particle of dark matter fulfils the first law of motion, to match the speed and mass of the particle at $\mathrm{B}$ in figure $2 \mathrm{a}$ :

The force at point $\mathrm{B}$, fig2a is $\boldsymbol{f}=\boldsymbol{m g}=\mathbf{1} \times \mathbf{2}=\mathbf{2}$,

The force at point A, fig2b is: $\boldsymbol{f}=\boldsymbol{m} \boldsymbol{g}=\mathbf{1} \times \mathbf{1}=\mathbf{1}$, and when this force is added as: $\mathbf{2} \times \boldsymbol{p o i n t} \boldsymbol{A}$ the answer is $\boldsymbol{f}=\mathbf{2 m \boldsymbol { g }}$. this shows that both the points $\mathrm{A}$ at fig $2 \mathrm{~b}$ balances point $\mathrm{B}$ at fig2a. This balance is made possible by the supportive law of motion, lest the nucleus would not form as balance would not be possible.

Table 3. The motion of dark matter and anti-dark matter

\begin{tabular}{|c|c|}
\hline From dark matter to nucleus of matter - fig2a & From anti-dark matter to nucleus of antimatter - fig2b \\
\hline 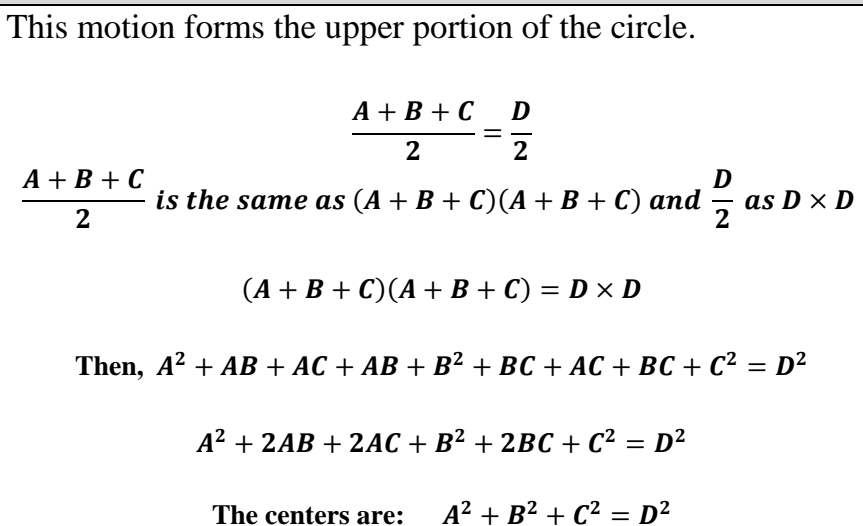 & $\begin{array}{l}\text { This motion forms the lower portion of the circle. } \\
\qquad \begin{array}{l}\frac{A+B}{2}=\frac{C}{2} \\
\frac{A+B}{2} \text { this is the same as, }(A+B)(A+B) \text { and } \frac{C}{2}=C \times C \\
(A+B)(A+B)=C \times C \\
\text { then, } \quad A^{2}+2 A B+B^{2}=C^{2} \\
\text { The centres are: } \quad A^{2}+B^{2}=C^{2}\end{array}\end{array}$ \\
\hline
\end{tabular}




\section{Fig3 Solar wave}

Fig3a. particles of the solar level

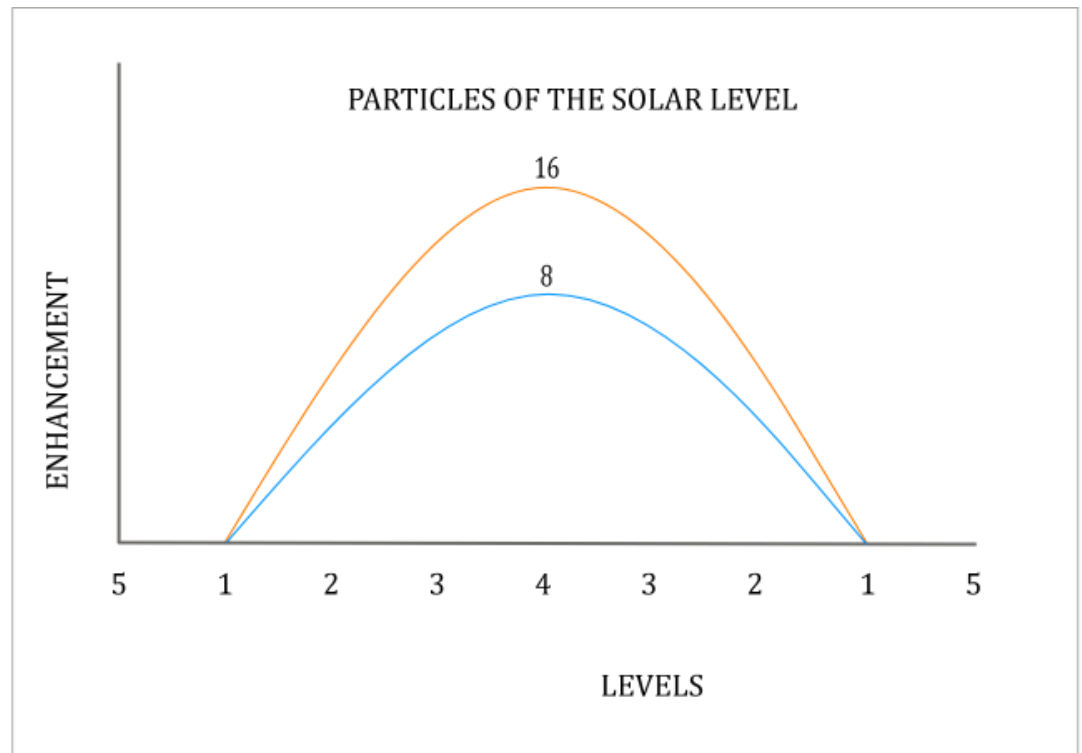

IV. NUCLEAR WAVES

Table 4. formation of the solar level

Fig3b. antiparticles of the solar level

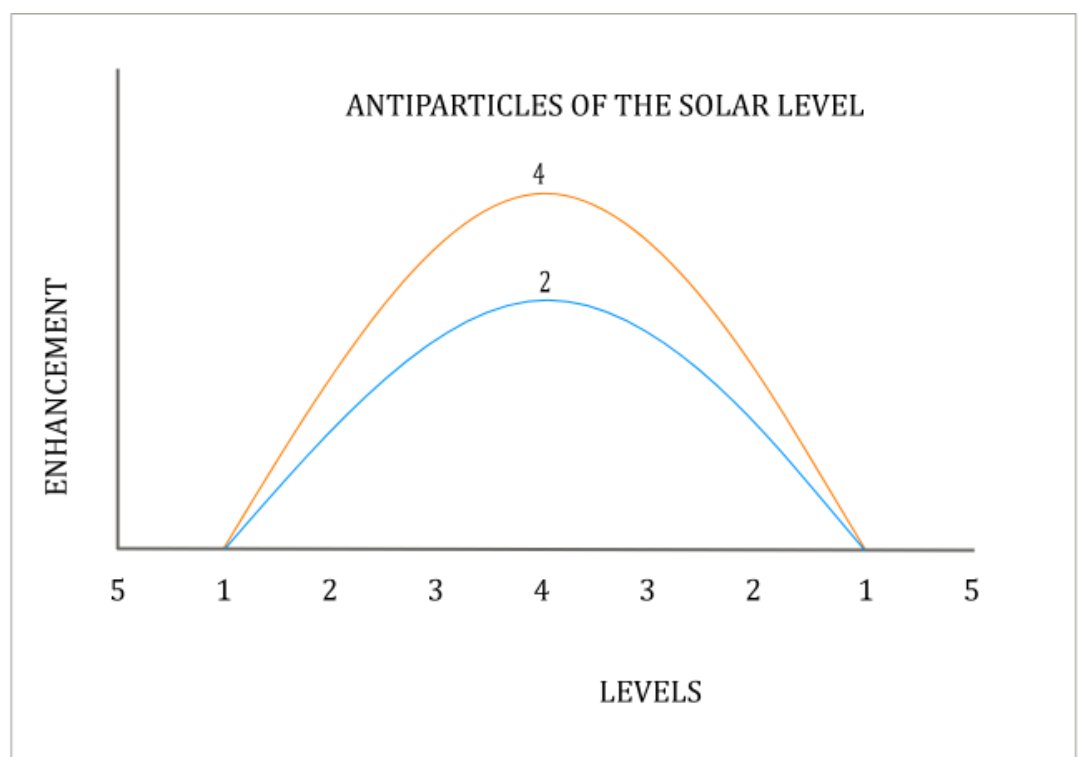

\begin{tabular}{|c|c|}
\hline Matter & Antimatter \\
\hline $\begin{array}{l}\text { Wave development }-n=0: \\
E=m_{1} \times m_{2} \\
\text { And therefore, } E=\frac{1}{4} \times \frac{1}{4}=\frac{1}{16} \text { (taken from the formation of the nucleus of matter). } \\
N=\frac{1}{4} E \\
\text { Then, } \\
N=\frac{1}{4} \times \frac{1}{16}=\frac{1}{64} \text { (this is the nucleus formed) } \\
\text { The radius of this nucleus is a proton }\end{array}$ & $\begin{array}{l}\text { Wave development }-n=0: \\
E=m_{1} \times m_{2} \\
\text { And therefore, } E=\frac{1}{2} \times \frac{1}{2}=\frac{1}{4} \text { (taken from the formation of the antimatter nucleus). } \\
N=\frac{1}{2} E \\
\text { Then, } \\
N=\frac{1}{2} \times \frac{1}{4}=\frac{1}{8} \text { (this is the nucleus formed) } \\
\text { The radius of this nucleus is a proton }\end{array}$ \\
\hline
\end{tabular}

This publication is licensed under Creative Commons Attribution CC BY. 
$r=\sqrt{N}$
And therefore, $r=\sqrt{\frac{1}{64}}$ which means $r=\frac{1}{8}$

The proton decays into an electron and the electron works much harder to balance the third wave of dark matter. Hence its force yields additional energy to add on the strength of the energy produced by the neutron and proton in the core.

$\boldsymbol{E}_{2}=\mathbf{4 r}$ (The electron energy working against the third wave of dark matter) And therefore, $E_{2}=4 \times \frac{1}{8}=\frac{1}{2}$

Solar level -- $n=1$ :

$E=m_{1} \times m_{2}$

Then $P=\frac{1}{2} \times \frac{1}{2}=\frac{1}{4}$

And $N=\frac{1}{4} E$,

Then $N=\frac{1}{4} \times \frac{1}{4}=\frac{1}{16}$

The radius $r=\sqrt{\frac{1}{16}}=\frac{1}{4}$

$\boldsymbol{E}_{2}=\mathbf{4 r}$ (The electron energy working against the third wave of dark matter) And therefore, $E_{2}=4 \times \frac{1}{4}=1$

Solar level -- $n=2$ :

$E=m_{1} \times m_{2}$

Then $P=1 \times 1=1$

And $N=\frac{1}{4} \times E$

Then $\mathbf{N}=\frac{1}{4} \times \mathbf{1}=\frac{1}{4}$

The radius $\mathbf{r}=\sqrt{\frac{1}{4}}=\frac{1}{2}$

$\boldsymbol{E}_{2}=\mathbf{4 r}$ (The electron energy working against the third wave of dark matter) And therefore, $E_{2}=4 \times \frac{1}{2}=2$
$r=\sqrt{N}$

And therefore, $r=\sqrt{\frac{1}{8}}$ which means $r=\frac{1}{2} \sqrt{\frac{1}{2}}$

The proton decays into an electron and the electron works much harder to balance the third wave of dark matter. Hence its force yields additional energy to add on the strength of the energy produced by the neutron and proton in the core.

$\boldsymbol{E}_{2}=2 \boldsymbol{r}$ (The positron energy working against the second wave of dark matter)

And therefore, $E_{2}=2 \times \frac{1}{2} \sqrt{\frac{1}{2}}=\sqrt{\frac{1}{2}}$

Solar level -- $n=1$ :

$E=m_{1} \times m_{2}$

Then $P=\sqrt{\frac{1}{2}} \times \sqrt{\frac{1}{2}}=\frac{1}{2}$

And $N=\frac{1}{2} E$,

Then $N=\frac{1}{2} \times \frac{1}{2}=\frac{1}{4}$

The radius $r=\sqrt{\frac{1}{4}}=\frac{1}{2}$

$\boldsymbol{E}_{2}=2 \boldsymbol{r}$ (The positron energy working against the second wave of dark matter) And therefore, $E_{2}=2 \times \frac{1}{2}=1$

Solar level -- $n=2$

$E=m_{1} \times m_{2}$

Then $P=\mathbf{1} \times \mathbf{1}=\mathbf{1}$

And $N=\frac{1}{4} \times E$,

Then $\mathrm{N}=\frac{1}{4} \times 1=\frac{1}{4}$

The radius $r=\sqrt{\frac{1}{4}}=\frac{1}{2}$

$E_{2}=2 r$ (The electron energy working against the third wave of dark matter)

And therefore, $E_{2}=2 \times \frac{1}{2}=1$ 


\section{Solar level -- $n=3$ :}

Every time the second law of motion is at work, the supportive law of motion is activated: $E_{\text {support }}=2 E_{\text {applied }}$

Meaning, $\boldsymbol{E}_{\text {support }}=\mathbf{2} \boldsymbol{E}_{2}$

And therefore, $E_{\text {support }}=2 \times 2=4$

\begin{tabular}{|c|c|}
\hline Supporting wave & Supported wave \\
\hline $\begin{array}{l}\text { Solar level }-n=4: \\
E=m_{1} \times m_{2}\end{array}$ & $\begin{array}{l}\text { Solar level -- } n=4: \\
E=m_{1} \times m_{2}\end{array}$ \\
\hline Then, $P=\mathbf{4} \times \mathbf{4}=16$ & Then, $P=2 \times 2=4$ \\
\hline Here, $\boldsymbol{N}=\boldsymbol{E}$ because nuclear enhancement & Here, $\boldsymbol{N}=\boldsymbol{E}$ because nuclear enhancement \\
\hline has now been able to balance with $\boldsymbol{M}=\frac{\mathbf{1}}{\mathbf{4}}$ & has now been able to balance with $\boldsymbol{M}=\frac{\mathbf{1}}{\mathbf{4}}$ \\
\hline to a ratio of $1: 1$ speed. The action of the & to a ratio of $1: 1$ speed. The action of the \\
\hline nucleus is equal to the reaction of the third & nucleus is equal to the reaction of the third \\
\hline level of dark matter. & level of dark matter. \\
\hline Therefore, $\boldsymbol{N}=\mathbf{1} \times \boldsymbol{E}$, & Therefore, $\boldsymbol{N}=\mathbf{1} \times \boldsymbol{E}$, \\
\hline Then $\boldsymbol{N}=\mathbf{1 6}$ & Then $\boldsymbol{N}=\mathbf{4}$ \\
\hline The radius of this particle, $r=\sqrt{\mathbf{1 6}}=\mathbf{4}$ & The radius of this particle, $r=\sqrt{4}=2$ \\
\hline $\begin{array}{l}\boldsymbol{E}_{2}=\mathbf{4 r} \text { (The electron energy working } \\
\text { against the third wave of dark matter) }\end{array}$ & $\begin{array}{l}\boldsymbol{E}_{2}=\mathbf{4 r} \text { (The electron energy working } \\
\text { against the third wave of dark matter) }\end{array}$ \\
\hline And therefore, $E_{2}=4 \times 4=16$ & And therefore, $E_{2}=4 \times 2=8$ \\
\hline
\end{tabular}

Solar level -- $\boldsymbol{n}=\mathbf{3}$ :

Every time the second law of motion is at work, the supportive law of motion is activated: $E_{\text {support }}=2 E_{\text {applied }}$

Meaning, $\boldsymbol{E}_{\text {support }}=\mathbf{2} \boldsymbol{E}_{2}$

And therefore, $\boldsymbol{E}_{\text {support }}=2 \times 1=2$

\begin{tabular}{|c|c|}
\hline Supporting wave & Supported wave \\
\hline $\begin{array}{l}\text { Solar level -- } n=4: \\
E=m_{1} \times m_{2}\end{array}$ & $\begin{array}{l}\text { Solar level -- } n=4: \\
E=m_{1} \times m_{2}\end{array}$ \\
\hline Then, $P=2 \times 2=4$ & Then, $P=\mathbf{1} \times \mathbf{1}=\mathbf{1}$ \\
\hline Here, $\boldsymbol{N}=\boldsymbol{E}$ because nuclear enhancement & Here, $\boldsymbol{N}=\boldsymbol{E}$ because nuclear enhancement \\
\hline has now been able to balance with $\boldsymbol{M}=\frac{\mathbf{1}}{2}$ & has now been able to balance with $\boldsymbol{M}=\frac{\mathbf{1}}{2}$ \\
\hline to a ratio of $1: 1$ speed. The action of the & to a ratio of $1: 1$ speed. The action of the \\
\hline nucleus is equal to the reaction of the third & nucleus is equal to the reaction of the third \\
\hline level of dark matter. & level of dark matter. \\
\hline Therefore, $\boldsymbol{N}=\mathbf{1} \times \boldsymbol{E}$, & Therefore, $\boldsymbol{N}=\mathbf{1} \times \boldsymbol{E}$, \\
\hline Then $\boldsymbol{N}=\mathbf{4}$ & Then $\boldsymbol{N}=\mathbf{1}$ \\
\hline The radius of this particle, $r=\sqrt{4}=2$ & The radius of this particle, $\boldsymbol{r}=\sqrt{\mathbf{1}}=\mathbf{1}$ \\
\hline $\begin{array}{l}\boldsymbol{E}_{2}=\mathbf{2 r} \text { (The electron energy working } \\
\text { against the third wave of dark matter) } \\
\text { And therefore, } \boldsymbol{E}_{2}=\mathbf{2} \times \mathbf{2}=\mathbf{4}\end{array}$ & $\begin{array}{l}\boldsymbol{E}_{2}=2 \boldsymbol{r} \text { (The electron energy working } \\
\text { against the third wave of dark matter) } \\
\text { And therefore, } \boldsymbol{E}_{2}=\mathbf{2} \times \mathbf{1}=\mathbf{2}\end{array}$ \\
\hline
\end{tabular}




\section{GALACTIC NUCLEAR ENHANCEMENT - NUCLEUS OF MATTER}

\section{Fig4 galactic wave}

Fig4a. particles of the galactic level

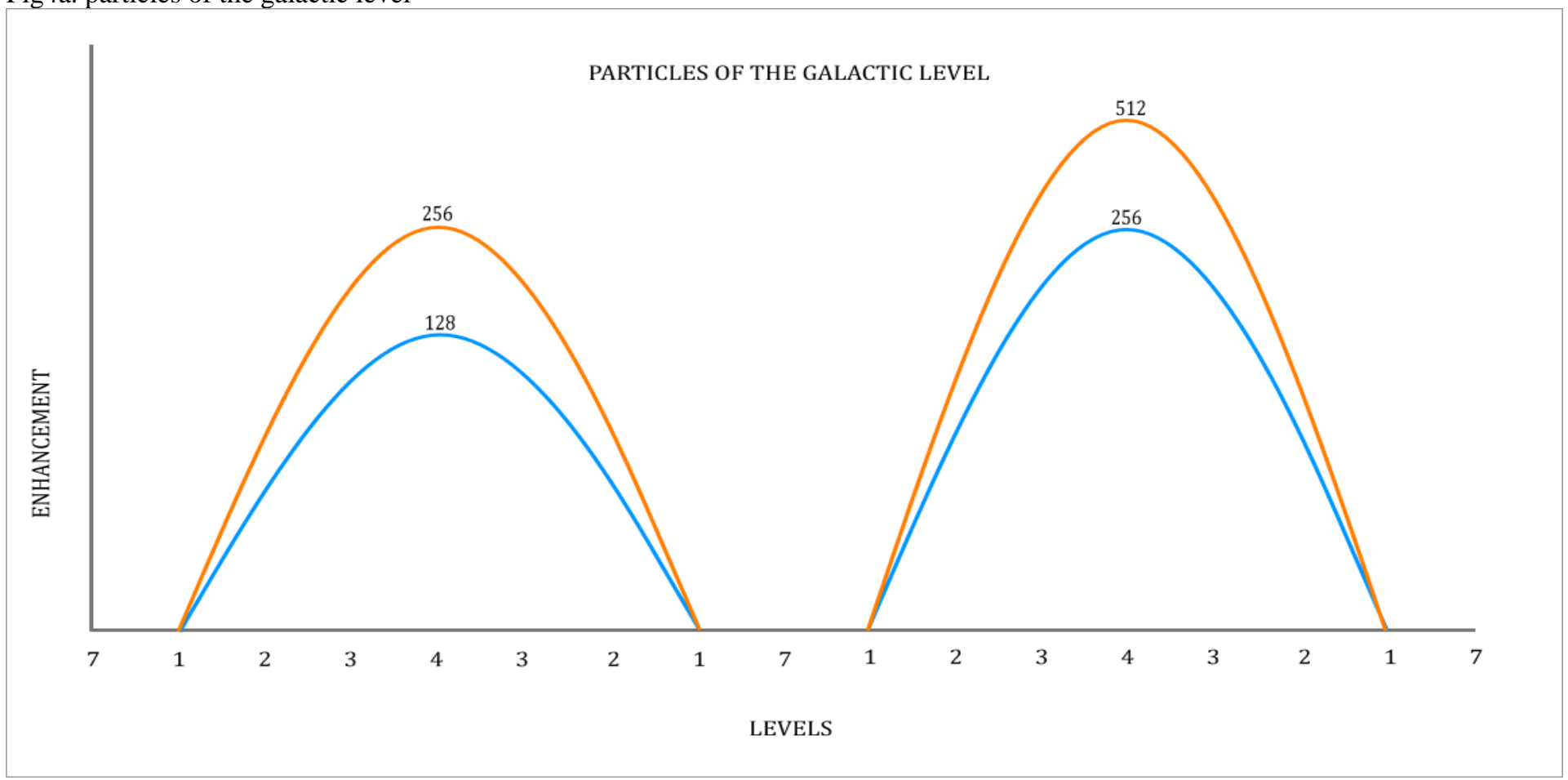

Table 5. formation of the Galactic level

\begin{tabular}{|c|c|}
\hline Matter & Matter \\
\hline $\begin{array}{l}\text { Galactic level }-n=7.1: \\
E=m_{1} \times m_{2} \\
\text { Then } E=16 \times 16=256\end{array}$ & $\begin{array}{l}\text { Galactic level }-n=7.1: \\
E=m_{1} \times m_{2} \\
\text { Then } E=8 \times 8=64\end{array}$ \\
\hline
\end{tabular}

This publication is licensed under Creative Commons Attribution CC BY. 
And $N=\frac{1}{4} E$
Then $N=\frac{1}{4} \times 256=64$

The radius $r=\sqrt{\mathbf{6 4}}=\mathbf{8}$

$\boldsymbol{E}_{2}=\mathbf{4 r}$ (The electron energy working against the third wave of dark matter)

And therefore, $E_{2}=\mathbf{4} \times \mathbf{8}=\mathbf{3 2}$

Galactic level $-n=7.2$ :

$E=m_{1} \times m_{2}$

Then $E=32 \times \mathbf{3 2}=\mathbf{1 0 2 4}$

And $N=\frac{1}{4} E$,

Then $N=\frac{1}{4} \times 1024=256$

The radius $r=\sqrt{\mathbf{2 5 6}}=\mathbf{1 6}$

$\boldsymbol{E}_{2}=\mathbf{4 r}$ (The electron energy working against the third wave of dark matter)

And therefore, $E_{2}=4 \times 16=64$

Galactic level $-\boldsymbol{n}=\mathbf{7 . 3}$ :

Every time the second law of motion is at work, the supportive law of motion is activated:

$E_{\text {support }}=2 E_{\text {applied }}$

Meaning, $\boldsymbol{E}_{\text {support }}=\mathbf{2} \boldsymbol{E}_{2}$

And therefore, $\boldsymbol{E}_{\text {support }}=\mathbf{2} \times \mathbf{6 4}=\mathbf{1 2 8}$

\begin{tabular}{|l|l|}
\hline & \\
\hline Galactic level $-\boldsymbol{n}=\mathbf{7 . 4 :}$ & Galactic level $-\boldsymbol{n}=\mathbf{7 . 4 :}$ \\
$\boldsymbol{E}=\boldsymbol{m}_{1} \times \boldsymbol{m}_{\mathbf{2}}$ & $\boldsymbol{E}=\boldsymbol{m}_{1} \times \boldsymbol{m}_{2}$ \\
Then, $\boldsymbol{P}=\mathbf{1 2 8} \times \mathbf{1 2 8}=\mathbf{1 6 3 8 4}$ & Then, $\boldsymbol{P}=\mathbf{6 4} \times \mathbf{6 4}=\mathbf{4 0 9 6}$ \\
\hline
\end{tabular}

And $N=\frac{1}{2} E$,

Then $N=\frac{1}{4} \times 64=16$

The radius $r=\sqrt{\mathbf{1 6}}=4$

$\boldsymbol{E}_{2}=\mathbf{4 r}$ (The electron energy working against the third wave of dark matter)

And therefore, $E_{2}=4 \times 4=16$

Galactic level $-n=7.2$ :

$E=m_{1} \times m_{2}$

Then $E=16 \times 16=256$

And $N=\frac{1}{4} E$,

Then $N=\frac{1}{4} \times 256=64$

The radius $r=\sqrt{\mathbf{6 4}}=\mathbf{8}$

$\boldsymbol{E}_{2}=\mathbf{4 r}$ (The electron energy working against the third wave of dark matter)

And therefore, $\boldsymbol{E}_{2}=\mathbf{4} \times \mathbf{8}=\mathbf{3 2}$

Galactic level $-\boldsymbol{n}=7.3$ :

Every time the second law of motion is at work, the supportive law of motion is activated:

$E_{\text {support }}=2 E_{\text {applied }}$

Meaning, $\boldsymbol{E}_{\text {support }}=\mathbf{2} \boldsymbol{E}_{2}$

And therefore, $E_{\text {support }}=2 \times 32=64$

\begin{tabular}{|l|l|}
\hline & \\
\hline Galactic level $-\boldsymbol{n}=\mathbf{7 . 4 :}$ & Galactic level $-\boldsymbol{n}=\mathbf{7 . 4 :}$ \\
$\boldsymbol{E}=\boldsymbol{m}_{\mathbf{1}} \times \boldsymbol{m}_{\mathbf{2}}$ & $E=\boldsymbol{m}_{1} \times \boldsymbol{m}_{2}$ \\
Then, $\boldsymbol{P}=\mathbf{6 4} \times \mathbf{6 4 = 4 0 9 6}$ & Then, $\boldsymbol{P}=\mathbf{3 2} \times \mathbf{3 2}=\mathbf{1 0 2 4}$ \\
\hline
\end{tabular}


has now been able to balance with $\boldsymbol{M}=\frac{1}{\mathbf{4}}$

to a ratio of 1:1 speed.

Therefore, $\boldsymbol{N}=\mathbf{1} \times \boldsymbol{E}$,

\section{Then $\boldsymbol{N}=\mathbf{1 6 3 8 4}$}

The radius, $r=\sqrt{\mathbf{1 6 3 8 4}}=\mathbf{1 2 8}$

$\boldsymbol{E}_{2}=\mathbf{4 r}$ (The electron energy working against the third wave of dark matter) And therefore, $E_{2}=\mathbf{4} \times \mathbf{1 2 8}=\mathbf{5 1 2}$ has now been able to balance with $\boldsymbol{M}=\frac{1}{\mathbf{4}}$

to a ratio of $1: 1$ speed.

Therefore, $\boldsymbol{N}=\mathbf{1} \times \boldsymbol{E}$,

Then $\boldsymbol{N}=\mathbf{4 0 9 6}$

The radius, $r=\sqrt{\mathbf{4 0 9 6}}=\mathbf{6 4}$

$\boldsymbol{E}_{2}=\mathbf{4 r}$ (The electron energy working against the third wave of dark matter)

And therefore, $E_{2}=4 \times 64=256$
Here, $\boldsymbol{N}=\boldsymbol{E}$ because nuclear

enhancement has now been able to

balance with $\boldsymbol{M}=\frac{\mathbf{1}}{\mathbf{4}}$ to a ratio of 1:1

speed.

Therefore, $\boldsymbol{N}=\mathbf{1} \times \boldsymbol{E}$,

Then $\boldsymbol{N}=\mathbf{4 0 9 6}$

The radius, $r=\sqrt{\mathbf{4 0 9 6}}=\mathbf{6 4}$

$\boldsymbol{E}_{2}=\mathbf{4 r}$ (The electron energy working against the third wave of dark matter)

And therefore, $E_{2}=4 \times 64=256$ enhancement has now been able to

balance with $\boldsymbol{M}=\frac{\mathbf{1}}{\mathbf{4}}$ to a ratio of $1: 1$

speed.

Therefore, $\boldsymbol{N}=\mathbf{1} \times \boldsymbol{E}$,

Then $\boldsymbol{N}=\mathbf{1 0 2 4}$

The radius, $r=\sqrt{\mathbf{1 0 2 4}}=\mathbf{3 2}$

$\boldsymbol{E}_{2}=\mathbf{4 r}$ (The electron energy working against the third wave of dark matter) And therefore, $E_{2}=4 \times 32=128$ 


\section{GALACTIC NUCLEAR ENHANCEMENT - NUCLEUS OF ANTIMATTER}

\section{Fig4 galactic wave}

Fig4b. antiparticles of the galactic leve

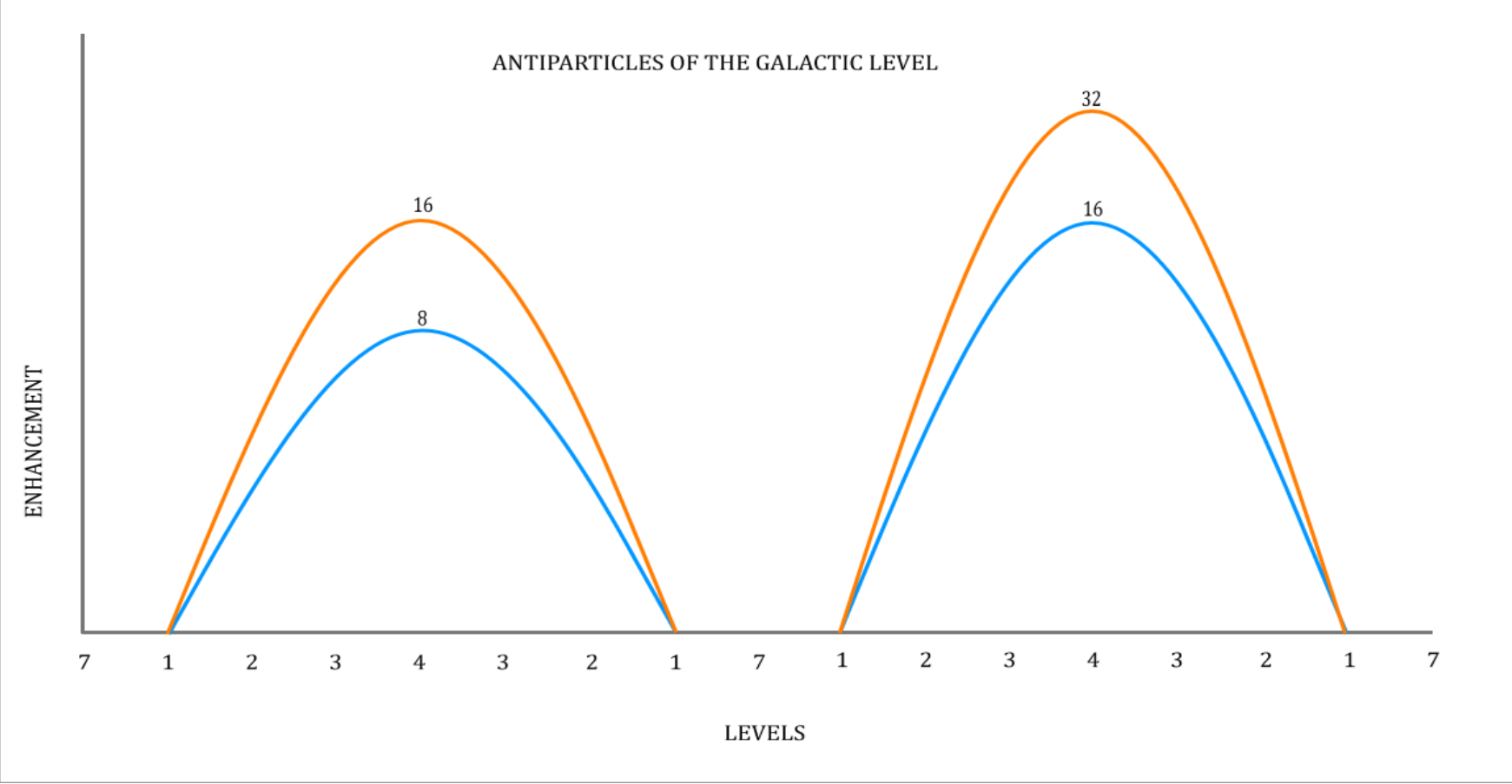

Table 5.1 formation of the antiparticles of the galactic level 
Galactic level $-\boldsymbol{n}=7.1$ :

$E=m_{1} \times m_{2}$

$E=m_{1} \times m_{2}$

Then $E=\mathbf{4} \times \mathbf{4}=\mathbf{1 6}$

And $N=\frac{1}{2} E$,

Then $N=\frac{1}{2} \times 16=8$

The radius $r=\sqrt{\mathbf{8}}=2 \sqrt{2}$

$\boldsymbol{E}_{2}=2 \boldsymbol{r}$ (The electron energy working against the third wave of dark matter)

And therefore, $E_{2}=2 \times 2 \sqrt{2}=4 \sqrt{2}$

Galactic level $-n=7.2$ :

$E=m_{1} \times m_{2}$

Then $E=4 \sqrt{2} \times 4 \sqrt{2}=32$

And $N=\frac{1}{2} E$,

Then $N=\frac{1}{2} \times 32=16$

The radius $r=\sqrt{\mathbf{1 6}}=\mathbf{4}$

$\boldsymbol{E}_{2}=2 \boldsymbol{r}$ (The positron energy working against the second wave of dark matter)

And therefore, $\boldsymbol{E}_{2}=\mathbf{2} \times \mathbf{4}=\mathbf{8}$

Galactic level $-\boldsymbol{n}=\mathbf{7 . 3}$ :

Every time the second law of motion is at work, the supportive law of motion is activated:

$E_{\text {support }}=2 E_{\text {applied }}$

Meaning, $\boldsymbol{E}_{\text {support }}=\mathbf{2} \boldsymbol{E}_{2}$

And therefore, $E_{\text {support }}=2 \times 8=16$

\begin{tabular}{|l|l|}
\hline & \\
\hline Galactic level $-\boldsymbol{n}=7.4:$ & Galactic level $-n=7.4:$ \\
\hline
\end{tabular}

Then $E=2 \times 2=4$

And $N=\frac{1}{2} E$,

Then $N=\frac{1}{2} \times \mathbf{4}=\mathbf{2}$

The radius $\boldsymbol{r}=\sqrt{\mathbf{2}}$

$\boldsymbol{E}_{2}=\mathbf{2 r}$ (The electron energy working against the third wave of dark matter)

And therefore, $\boldsymbol{E}_{2}=2 \times \mathbf{4}=\mathbf{2} \sqrt{2}$

Galactic level $-\boldsymbol{n}=7.2$ :

$E=m_{1} \times m_{2}$

Then $E=2 \sqrt{2} \times 2 \sqrt{2}=8$

And $N=\frac{1}{2} E$,

Then $\boldsymbol{N}=\frac{1}{\mathbf{2}} \times \mathbf{8}=\mathbf{4}$

The radius $r=\sqrt{\mathbf{4}}=2$

$\boldsymbol{E}_{2}=\mathbf{2 r}$ (The positron energy working against the third wave of dark matter)

And therefore, $E_{2}=2 \times 2=4$

Galactic level $-\boldsymbol{n}=7.3$ :

Every time the second law of motion is at work, the supportive law of motion is activated:

$E_{\text {support }}=2 E_{\text {applied }}$

Meaning, $\boldsymbol{E}_{\text {support }}=\mathbf{2} \boldsymbol{E}_{2}$

And therefore, $\boldsymbol{E}_{\text {support }}=\mathbf{2} \times \mathbf{4}=\mathbf{8}$

\begin{tabular}{|l|l|}
\hline & \\
\hline Galactic level $-n=7.4:$ & Galactic level $-n=7.4:$ \\
\hline
\end{tabular}




\section{$E=m_{1} \times m_{2}$ \\ Then, $P=\mathbf{8} \times \mathbf{8}=\mathbf{6 4}$}

Then, $P=\mathbf{1 6} \times \mathbf{1 6}=\mathbf{2 5 6}$

Here, $\boldsymbol{N}=\boldsymbol{E}$ because nuclear enhancement

Here, $\boldsymbol{N}=\boldsymbol{E}$ because nuclear enhancement

has now been able to balance with $\boldsymbol{M}=\frac{\mathbf{1}}{\mathbf{2}}$

to a ratio of $1: 1$ speed.

Therefore, $\boldsymbol{N}=\mathbf{1} \times \boldsymbol{E}$,

Then $\boldsymbol{N}=\mathbf{2 5 6}$

The radius, $r=\sqrt{\mathbf{2 5 6}}=\mathbf{1 6}$

$E_{2}=2 r$ (The electron energy working against the third wave of dark matter)

And therefore, $\boldsymbol{E}_{2}=\mathbf{2} \times \mathbf{1 6}=\mathbf{3 2}$ has now been able to balance with $\boldsymbol{M}=\frac{\mathbf{1}}{\mathbf{2}}$

to a ratio of $1: 1$ speed.

Therefore, $\boldsymbol{N}=\mathbf{1} \times \boldsymbol{E}$,

Then $\boldsymbol{N}=\mathbf{6 4}$

The radius, $r=\sqrt{\mathbf{6 4}}=8$

$\boldsymbol{E}_{2}=\mathbf{2 r}$ (The electron energy working against the third wave of dark matter) And therefore, $E_{2}=\mathbf{2} \times \mathbf{8}=\mathbf{1 6}$
$E=m_{1} \times m_{2}$

Then, $P=\mathbf{8} \times \mathbf{8}=\mathbf{6 4}$

Here, $\boldsymbol{N}=\boldsymbol{E}$ because nuclear

enhancement has now been able to

balance with $\boldsymbol{M}=\frac{\mathbf{1}}{2}$ to a ratio of 1:1

speed.

Therefore, $\boldsymbol{N}=\mathbf{1} \times \boldsymbol{E}$,

Then $\boldsymbol{N}=\mathbf{6 4}$

The radius, $r=\sqrt{\mathbf{6 4}}=\mathbf{8}$

$\boldsymbol{E}_{2}=\mathbf{2 r}$ (The electron energy working against the third wave of dark matter)

And therefore, $E_{2}=\mathbf{2} \times \mathbf{8}=\mathbf{1 6}$

\section{$E=m_{1} \times m_{2}$}

Then, $P=\mathbf{4} \times \mathbf{4}=\mathbf{1 6}$

Here, $\boldsymbol{N}=\boldsymbol{E}$ because nuclear

enhancement has now been able to

balance with $\boldsymbol{M}=\frac{\mathbf{1}}{\mathbf{2}}$ to a ratio of $1: 1$

speed.

Therefore, $\boldsymbol{N}=\mathbf{1} \times \boldsymbol{E}$,

Then $\boldsymbol{N}=\mathbf{1 6}$

The radius, $r=\sqrt{\mathbf{1 6}}=4$

$\boldsymbol{E}_{2}=\mathbf{2 r}$ (The electron energy working against the third wave of dark matter)

And therefore, $E_{2}=2 \times 4=8$ 


\section{UNIVERSAL NUCLEAR ENHANCEMENT - NUCLEUS OF MATTER}

Fig 5. Universal wave

Fig5a. formation of particle of the universal level.

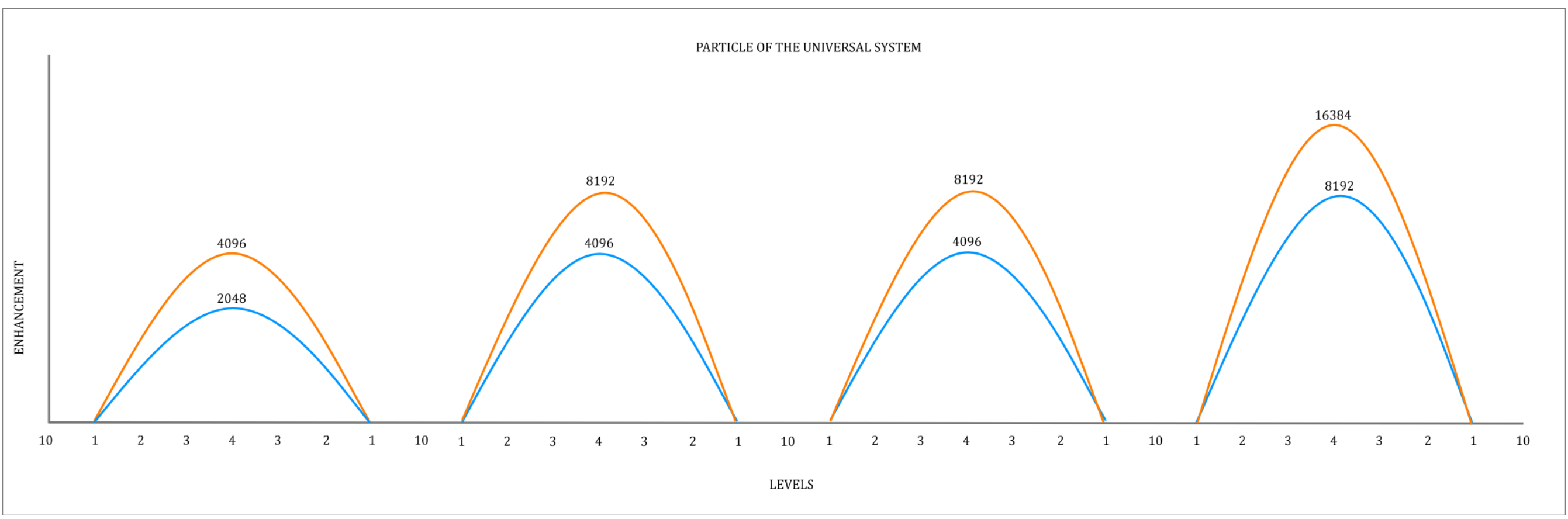

This publication is licensed under Creative Commons Attribution CC BY.

http://dx.doi.org/10.29322/IJSRP.11.10.2021.p11876 
Table 6. formation of particles of the universal level

\begin{tabular}{|c|c|}
\hline Particles & Particles \\
\hline Universal level $-n=8.1$ : & Universal level $-n=8.1$ : \\
\hline$E=m_{1} \times m_{2}$ & $E=m_{1} \times m_{2}$ \\
\hline Then $E=512 \times 512=\mathbf{2 6 2 1 4 4}$ & Then $E=256 \times 256=\mathbf{6 5 5 3 6}$ \\
\hline And $N=\frac{1}{4} E$, & And $N=\frac{1}{4} E$, \\
\hline Then $N=\frac{1}{4} \times 262144=65536$ & Then $N=\frac{1}{4} \times 65536=16384$ \\
\hline The radius $r=\sqrt{\mathbf{6 5 5 3 6}}=\mathbf{2 5 6}$ & The radius $r=\sqrt{\mathbf{1 6 3 8 4}}=128$ \\
\hline$E_{2}=4 r$ (The electron energy working against the third wave of dark matter) & $E_{2}=4 r($ The electron energy working against the third wave of dark matter) \\
\hline And therefore, $E_{2}=4 \times 256=1024$ & And therefore, $E_{2}=\mathbf{4} \times \mathbf{1 2 8}=\mathbf{5 1 2}$ \\
\hline $\begin{array}{l}\text { Universal level }-n=8.2 \text { : } \\
E=m_{1} \times m_{2}\end{array}$ & $\begin{array}{l}\text { Universal level }-n=8.2: \\
E=m_{1} \times m_{2}\end{array}$ \\
\hline Then $E=1024 \times 1024=1048576$ & Then $E=\mathbf{5 1 2} \times \mathbf{5 1 2}=\mathbf{2 6 2 1 4 4}$ \\
\hline And $N=\frac{1}{4} E$ & And $N=\frac{1}{4} E$ \\
\hline Then $N=\frac{1}{4} \times 1048576=262144$ & Then $N=\frac{1}{4} \times 262144=65536$ \\
\hline The radius $r=\sqrt{\mathbf{2 6 2 1 4 4}}=\mathbf{5 1 2}$ & The radius $r=\sqrt{\mathbf{6 5 5 3 6}}=\mathbf{2 5 6}$ \\
\hline$E_{2}=4 r$ (The electron energy working against the third wave of dark matter) & $E_{2}=4 r($ The electron energy working against the third wave of dark matter) \\
\hline And therefore, $E_{2}=4 \times 512=2048$ & And therefore, $E_{2}=4 \times 256=1024$ \\
\hline Universal level $-n=9$ : & Universal level $-\boldsymbol{n}=9$ : \\
\hline $\begin{array}{l}\text { Every time the second law of motion is at work, the supportive law of motion is activated: } \\
\boldsymbol{E}_{\text {support }}=\mathbf{2} \boldsymbol{E}_{\text {applied }}\end{array}$ & $\begin{array}{l}\text { Every time the second law of motion is at work, the supportive law of motion is activated: } \\
\boldsymbol{E}_{\text {support }}=\mathbf{2} \boldsymbol{E}_{\text {applied }}\end{array}$ \\
\hline Meaning, $E_{\text {support }}=\mathbf{2} E_{2}$ & Meaning, $E_{\text {support }}=\mathbf{2} E_{2}$ \\
\hline And therefore, $E_{\text {support }}=2 \times 2048=4096$ & And therefore, $E_{\text {support }}=2 \times 1024=2048$ \\
\hline
\end{tabular}




\begin{tabular}{|c|c|}
\hline \multicolumn{2}{|l|}{ Universal level $-n=10$ : } \\
\hline$E=m_{1} \times m_{2}$ & $E=m_{1} \times m_{2}$ \\
\hline Then, $P=4096 \times 4096=16777216$ & Then, $P=2048 \times 2048=4194304$ \\
\hline Here, $\boldsymbol{N}=\boldsymbol{E}$ because nuclear enhancement & Here, $\boldsymbol{N}=\boldsymbol{E}$ because nuclear enhancement \\
\hline has now been able to balance with $\boldsymbol{M}=\frac{\mathbf{1}}{\mathbf{4}}$ & has now been able to balance with $\boldsymbol{M}=\frac{\mathbf{1}}{\mathbf{4}}$ \\
\hline to a ratio of $1: 1$ speed. & to a ratio of $1: 1$ speed. \\
\hline Therefore, $\boldsymbol{N}=\mathbf{1} \times \boldsymbol{E}$, & Therefore, $\boldsymbol{N}=\mathbf{1} \times \boldsymbol{E}$, \\
\hline Then $\boldsymbol{N}=\mathbf{1 6 7 7 7 2 1 6}$ & Then $N=\mathbf{4 1 9 4 3 0 4}$ \\
\hline The radius, $r=\sqrt{16777216}=4096$ & The radius, $r=\sqrt{4194304}=2048$ \\
\hline $\begin{array}{l}\boldsymbol{E}_{2}=\mathbf{4 r} \text { (The electron energy working } \\
\text { against the third wave of dark matter) }\end{array}$ & $\begin{array}{l}\boldsymbol{E}_{2}=\mathbf{4 r} \text { (The electron energy working } \\
\text { against the third wave of dark matter) }\end{array}$ \\
\hline And therefore, $E_{2}=4 \times 4096=16384$ & And therefore, $E_{2}=4 \times 2048=8192$ \\
\hline
\end{tabular}

Universal level $-\boldsymbol{n}=10$ :

$E$
$E=m_{1} \times m_{2}$

Then, $P=\mathbf{2 0 4 8} \times \mathbf{2 0 4 8}=\mathbf{4 1 9 4 3 0 4}$

Here, $\boldsymbol{N}=\boldsymbol{E}$ because nuclear

enhancement has now been able to

balance with $\boldsymbol{M}=\frac{1}{4}$ to a ratio of $1: 1$

speed.

Therefore, $\boldsymbol{N}=\mathbf{1} \times \boldsymbol{E}$,

Then $\boldsymbol{N}=\mathbf{4 1 9 4 3 0 4}$

The radius, $\boldsymbol{r}=\sqrt{\mathbf{4 1 9 4 3 0 4}}=\mathbf{2 0 4 8}$

$\boldsymbol{E}_{2}=\mathbf{4 r}$ (The electron energy working against the third wave of dark matter)

And therefore, $E_{2}=4 \times 2048=8192$
$E=m_{1} \times m_{2}$

Then, $P=\mathbf{1 0 2 4} \times \mathbf{1 0 2 4}=\mathbf{1 0 4 8 5 7 6}$

Here, $\boldsymbol{N}=\boldsymbol{E}$ because nuclear

enhancement has now been able to

balance with $\boldsymbol{M}=\frac{1}{4}$ to a ratio of $1: 1$ speed.

Therefore, $\boldsymbol{N}=\mathbf{1} \times \boldsymbol{E}$,

Then $\boldsymbol{N}=\mathbf{1 0 4 8 5 7 6}$

The radius, $\boldsymbol{r}=\sqrt{\mathbf{1 0 4 8 5 7 6}}=\mathbf{1 0 2 4}$

$\boldsymbol{E}_{2}=\mathbf{4 r}$ (The electron energy working against the third wave of dark matter)

And therefore, $\boldsymbol{E}_{2}=\mathbf{4} \times \mathbf{1 0 2 4}=\mathbf{4 0 9 6}$

\section{Particles}

\section{Universal level $-\boldsymbol{n}=8.1$ :}

$E=m_{1} \times m_{2}$

Then $E=256 \times 256=65536$

And $N=\frac{1}{4} E$,

Then $N=\frac{1}{4} \times 65536=16384$

The radius $r=\sqrt{\mathbf{1 6 3 8 4}}=\mathbf{1 2 8}$

$$
\begin{aligned}
& \text { Universal level }-\boldsymbol{n}=8.1 \text { : } \\
& E=m_{1} \times m_{2} \\
& \text { Then } E=128 \times 128=16384 \\
& \text { And } N=\frac{1}{4} E \text {, } \\
& \text { Then } N=\frac{1}{4} \times 16384=4096 \\
& \text { The radius } \boldsymbol{r}=\sqrt{\mathbf{4 0 9 6}}=\mathbf{6 4}
\end{aligned}
$$

This publication is licensed under Creative Commons Attribution CC BY. 
Universal level $-\boldsymbol{n}=8.2$ :

$E=m_{1} \times m_{2}$

Then $E=512 \times 512=\mathbf{2 6 2 1 4 4}$

And $N=\frac{1}{4} E$,

Then $N=\frac{1}{4} \times 262144=\mathbf{6 5 5 3 6}$

The radius $r=\sqrt{\mathbf{6 5 5 3 6}}=\mathbf{2 5 6}$

$\boldsymbol{E}_{2}=\mathbf{4 r}$ (The electron energy working against the third wave of dark matter)

And therefore, $E_{2}=4 \times 256=1024$

Universal level $-\boldsymbol{n}=9$ :

Every time the second law of motion is at work, the supportive law of motion is activated:

$E_{\text {support }}=2 E_{\text {applied }}$

Meaning, $E_{\text {support }}=2 E_{2}$

And therefore, $\boldsymbol{E}_{\text {support }}=\mathbf{2} \times \mathbf{1 0 2 4}=\mathbf{2 0 4 8}$

\section{Universal level $-\boldsymbol{n}=10$ :}

\begin{tabular}{l|l}
\hline $\boldsymbol{E}=\boldsymbol{m}_{\mathbf{1}} \times \boldsymbol{m}_{\mathbf{2}}$ & $\boldsymbol{E}=\boldsymbol{m}_{\mathbf{1}} \times \boldsymbol{m}_{\mathbf{2}}$ \\
Then, $\boldsymbol{P}=\mathbf{2 0 4 8} \times \mathbf{2 0 4 8}=\mathbf{4 1 9 4 3 0 4}$ & $\begin{array}{l}\text { Then, } \boldsymbol{P}=\mathbf{1 0 2 4} \times \mathbf{1 0 2 4}=\mathbf{1 0 4 8 5 7 6} \\
\text { Here, } \boldsymbol{N}=\boldsymbol{E} \text { because nuclear } \\
\text { Here, } \boldsymbol{N}=\boldsymbol{E} \text { because nuclear } \\
\text { enhancement has now been able to } \\
\text { enhancement has now been able to } \\
\text { balance with } \boldsymbol{M}=\frac{\mathbf{1}}{\mathbf{4}} \text { to a ratio of 1:1 } \\
\text { speed. }\end{array} \quad \begin{array}{l}\text { balance with } \boldsymbol{M}=\frac{\mathbf{1}}{\mathbf{4}} \text { to a ratio of } 1: 1 \\
\text { sheed. } \\
\text { Therefore, } \boldsymbol{N}=\mathbf{1} \times \boldsymbol{E},\end{array}$ \\
\hline
\end{tabular}

Universal level $-n=8.2$ :

$E=m_{1} \times m_{2}$

Then $E=\mathbf{2 5 6} \times \mathbf{2 5 6}=\mathbf{6 5 5 3 6}$

And $N=\frac{1}{4} E$,

Then $N=\frac{1}{4} \times 165536=16384$

The radius $r=\sqrt{\mathbf{1 6 3 8 4}}=\mathbf{1 2 8}$

$\boldsymbol{E}_{2}=\mathbf{4 r}$ (The electron energy working against the third wave of dark matter)

And therefore, $\boldsymbol{E}_{2}=\mathbf{4} \times \mathbf{1 2 8}=\mathbf{5 1 2}$

Universal level $-\boldsymbol{n}=9$ :

Every time the second law of motion is at work, the supportive law of motion is activated $E_{\text {support }}=2 E_{\text {applied }}$

Meaning, $\boldsymbol{E}_{\text {support }}=\mathbf{2} \boldsymbol{E}_{2}$

And therefore, $\boldsymbol{E}_{\text {support }}=\mathbf{2} \times \mathbf{5 1 2}=\mathbf{1 0 2 4}$

\section{Universal level $-\boldsymbol{n}=10$ :}

$E=\boldsymbol{m}_{1} \times \boldsymbol{m}_{2}$
Then, $P=\mathbf{1 0 2 4} \times \mathbf{1 0 2 4}=\mathbf{1 0 4 8 5 7 6}$

\section{$E=m_{1} \times m_{2}$}

Here, $\boldsymbol{N}=\boldsymbol{E}$ because nuclear

Then, $P=\mathbf{5 1 2} \times \mathbf{5 1 2}=\mathbf{2 6 2 1 4 4}$

enhancement has now been able to

Here, $\boldsymbol{N}=\boldsymbol{E}$ because nuclear

balance with $\boldsymbol{M}=\frac{1}{4}$ to a ratio of 1:1

enhancement has now been able to

speed.

Therefore, $\boldsymbol{N}=\mathbf{1} \times \boldsymbol{E}$, balance with $\boldsymbol{M}=\frac{1}{4}$ to a ratio of $1: 1$ speed.

Therefore, $\boldsymbol{N}=\mathbf{1} \times \boldsymbol{E}$, 


\begin{tabular}{|c|c|c|c|}
\hline Then $N=4194304$ & Then $N=1048576$ & Then $N=1048576$ & Then $N=262144$ \\
\hline The radius, $r=\sqrt{4194304}=2048$ & The radius, $r=\sqrt{1048576}=1024$ & The radius, $r=\sqrt{1048576}=1024$ & The radius, $r=\sqrt{262144}=512$ \\
\hline $\begin{array}{l}\boldsymbol{E}_{2}=\mathbf{4 r} \text { (The electron energy working } \\
\text { against the third wave of dark matter) }\end{array}$ & $\begin{array}{l}\boldsymbol{E}_{2}=\mathbf{4 r} \text { (The electron energy working } \\
\text { against the third wave of dark matter) }\end{array}$ & $\begin{array}{l}\boldsymbol{E}_{2}=\mathbf{4 r} \text { (The electron energy working } \\
\text { against the third wave of dark matter) }\end{array}$ & $\begin{array}{l}\boldsymbol{E}_{2}=\mathbf{4 r} \text { (The electron energy working } \\
\text { against the third wave of dark matter) }\end{array}$ \\
\hline And therefore, $E_{2}=4 \times 2048=8192$ & And therefore, $E_{2}=4 \times 1024=4096$ & And therefore, $E_{2}=4 \times 1024=4096$ & And therefore, $E_{2}=4 \times 512=2048$ \\
\hline
\end{tabular}

\section{UNIVERSAL NUCLEAR ENHANCEMENT - NUCLEUS OF ANTIMATTER}

Fig 5 b. formation of antiparticles of the universal level 
ANTIPARTICLE OF THE UNIVERSAL SYSTEM

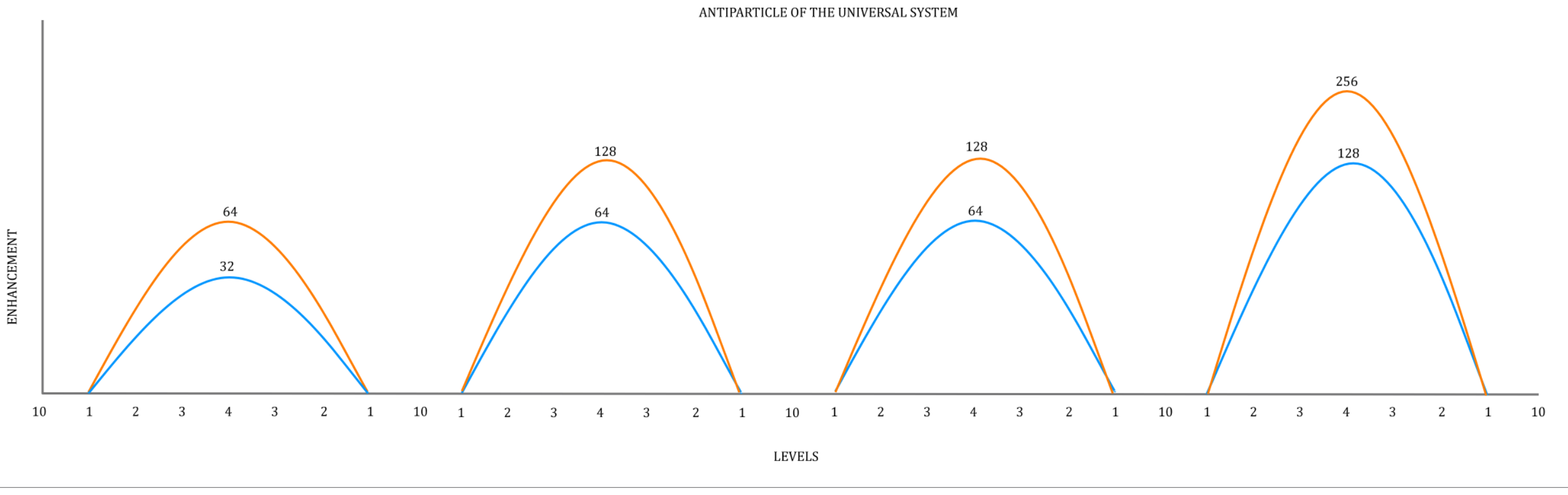

Table $6 \mathrm{~b}$. formation of the antiparticle of the universal level

\begin{tabular}{|c|c|}
\hline Antiparticles & Antiparticles \\
\hline Universal level $-n=8.1$ : & Universal level $-n=8.1$ : \\
\hline$E=m_{1} \times m_{2}$ & $E=m_{1} \times m_{2}$ \\
\hline Then $E=32 \times 32=1024$ & Then $E=16 \times 16=256$ \\
\hline And $N=\frac{1}{2} E$ & And $N=\frac{1}{2} E$, \\
\hline Then $N=\frac{1}{2} \times 1024=512$ & Then $N=\frac{1}{2} \times 256=128$ \\
\hline The radius $r=\sqrt{512}=16 \sqrt{2}$ & The radius $r=\sqrt{\mathbf{1 2 8}}=\mathbf{8} \sqrt{\mathbf{2}}$ \\
\hline $\boldsymbol{E}_{2}=2 \boldsymbol{r}($ The positron energy working against the third wave of dark matter $)$ & $E_{2}=2 \boldsymbol{r}$ (The positron energy working against the third wave of dark matter) \\
\hline And therefore, $E_{2}=2 \times 16 \sqrt{2}=32 \sqrt{2}$ & And therefore, $E_{2}=2 \times 8 \sqrt{2}=16 \sqrt{2}$ \\
\hline
\end{tabular}

This publication is licensed under Creative Commons Attribution CC BY. 
Universal level $-\boldsymbol{n}=8.2$ :

$E=m_{1} \times m_{2}$

Then $E=32 \sqrt{2} \times 32 \sqrt{2}=2048$

And $N=\frac{1}{2} E$,

Then $N=\frac{1}{2} \times 2048=1024$

The radius $\boldsymbol{r}=\sqrt{\mathbf{1 0 2 4}}=\mathbf{3 2}$

$\boldsymbol{E}_{2}=\mathbf{2 r}$ (The positron energy working against the third wave of dark matter)

And therefore, $E_{2}=2 \times 32=64$

Universal level $-\boldsymbol{n}=9$ :

Every time the second law of motion is at work, the supportive law of motion is activated:

$E_{\text {support }}=2 E_{\text {applied }}$

Meaning, $E_{\text {support }}=\mathbf{2} E_{2}$

And therefore, $E_{\text {support }}=2 \times 64=128$

Universal level $-\boldsymbol{n}=10$ :

\section{$E=m_{1} \times m_{2}$}

Then, $P=\mathbf{1 2 8} \times \mathbf{1 2 8}=\mathbf{1 6 3 8 4}$

Here, $\boldsymbol{N}=\boldsymbol{E}$ because nuclear

enhancement has now been able to

balance with $\boldsymbol{M}=\frac{\mathbf{1}}{\mathbf{2}}$ to a ratio of $1: 1$

speed.

Therefore, $\boldsymbol{N}=\mathbf{1} \times \boldsymbol{E}$

Then $\boldsymbol{N}=\mathbf{1 6 3 8 4}$

The radius, $\boldsymbol{r}=\sqrt{\mathbf{1 6 3 8 4}}=\mathbf{1 2 8}$

\section{$E=m_{1} \times m_{2}$}

Then, $P=\mathbf{6 4} \times \mathbf{6 4}=\mathbf{4 0 9 6}$

Here, $\boldsymbol{N}=\boldsymbol{E}$ because nuclear

enhancement has now been able to

balance with $\boldsymbol{M}=\frac{\mathbf{1}}{\mathbf{2}}$ to a ratio of $1: 1$

speed.

Therefore, $\boldsymbol{N}=\mathbf{1} \times \boldsymbol{E}$,

Then $\boldsymbol{N}=\mathbf{4 0 9 6}$

The radius, $r=\sqrt{\mathbf{4 0 9 6}}=\mathbf{6 4}$
Universal level $-\boldsymbol{n}=8.2$ :

$E=m_{1} \times m_{2}$

Then $E=16 \sqrt{2} \times 16 \sqrt{2}=512$

And $N=\frac{1}{2} E$,

Then $N=\frac{1}{2} \times 512=256$

The radius $r=\sqrt{\mathbf{2 5 6}}=\mathbf{1 6}$

$\boldsymbol{E}_{2}=\mathbf{4 r}$ (The electron energy working against the third wave of dark matter)

And therefore, $E_{2}=2 \times 16=32$

Universal level $-\boldsymbol{n}=9$ :

Every time the second law of motion is at work, the supportive law of motion is activated:

$E_{\text {support }}=2 E_{\text {applied }}$

Meaning, $\boldsymbol{E}_{\text {support }}=\mathbf{2} \boldsymbol{E}_{2}$

And therefore, $E_{\text {support }}=2 \times 32=64$

Universal level $-n=10$ :

$E=m_{1} \times m_{2}$

Then, $P=\mathbf{6 4} \times \mathbf{6 4}=\mathbf{4 0 9 6}$

$E=m_{1} \times m_{2}$

Here, $\boldsymbol{N}=\boldsymbol{E}$ because nuclear

enhancement has now been able to

balance with $\boldsymbol{M}=\frac{1}{2}$ to a ratio of $1: 1$

speed.

Therefore, $\boldsymbol{N}=\mathbf{1} \times \boldsymbol{E}$,

Then $N=4096$

The radius, $r=\sqrt{\mathbf{4 0 9 6}}=\mathbf{6 4}$
Then, $P=\mathbf{3 2} \times \mathbf{3 2}=\mathbf{1 0 2 4}$

Here, $\boldsymbol{N}=\boldsymbol{E}$ because nuclear

enhancement has now been able to balance with $\boldsymbol{M}=\frac{1}{2}$ to a ratio of 1:1 speed.

Therefore, $\boldsymbol{N}=\mathbf{1} \times \boldsymbol{E}$

Then $\boldsymbol{N}=\mathbf{1 0 2 4}$

The radius, $r=\sqrt{\mathbf{1 0 2 4}}=\mathbf{3 2}$ 


\begin{tabular}{|l|l|}
\hline $\begin{array}{l}\boldsymbol{E}_{2}=\mathbf{2 r}(\text { The positron energy working } \\
\text { against the third wave of dark matter) }\end{array}$ & $\begin{array}{l}\boldsymbol{E}_{2}=\mathbf{2 r} \text { (The positron energy working } \\
\text { against the third wave of dark matter) } \\
\text { And therefore, } \boldsymbol{E}_{\mathbf{2}}=\mathbf{2} \times \mathbf{1 2 8}=\mathbf{2 5 6}\end{array}$ \\
And therefore, $\boldsymbol{E}_{\mathbf{2}}=\mathbf{2} \times \mathbf{6 4}=\mathbf{1 2 8}$
\end{tabular}

$\boldsymbol{E}_{2}=\mathbf{2 r}$ (The positron energy working

against the third wave of dark matter)

$\boldsymbol{E}_{\mathbf{2}}=\mathbf{2 r}$ (The electron energy working against the third wave of dark matter)

And therefore, $E_{2}=2 \times 64=128$

\section{Antiparticles}

\section{Universal level $-\boldsymbol{n}=8.1$ :}

$E=m_{1} \times m_{2}$

Then $E=16 \times 16=256$

And $N=\frac{1}{2} E$,

Then $N=\frac{1}{2} \times 256=128$

The radius $r=\sqrt{\mathbf{1 2 8}}=\mathbf{8} \sqrt{\mathbf{2}}$

$\boldsymbol{E}_{2}=2 \boldsymbol{r}$ (The positron energy working against the third wave of dark matter)

And therefore, $E_{2}=2 \times 8 \sqrt{2}=16 \sqrt{2}$

Universal level $-\boldsymbol{n}=8.2$ :

$E=m_{1} \times m_{2}$

Then $E=16 \sqrt{2} \times 16 \sqrt{2}=512$

And $N=\frac{1}{2} E$,

Then $N=\frac{1}{2} \times 512=256$

The radius $r=\sqrt{\mathbf{2 5 6}}=\mathbf{1 6}$

$\boldsymbol{E}_{2}=\mathbf{4 r}$ (The positron energy working against the third wave of dark matter)

And therefore, $E_{2}=2 \times 16=32$

Universal level $-\boldsymbol{n}=9$ :

\section{Universal level $-\boldsymbol{n}=\mathbf{8} .1$ :}

$E=m_{1} \times m_{2}$

Then $\boldsymbol{E}=\mathbf{8} \times \mathbf{8}=\mathbf{6 4}$

And $N=\frac{1}{2} E$,

Then $N=\frac{1}{2} \times 64=32$

The radius $r=\sqrt{32}=\mathbf{4} \sqrt{2}$

$\boldsymbol{E}_{2}=2 \boldsymbol{r}$ (The positron energy working against the third wave of dark matter)

And therefore, $E_{2}=2 \times 4 \sqrt{2}=8 \sqrt{2}$

Universal level $-\boldsymbol{n}=8.2$ :

$E=m_{1} \times m_{2}$

Then $E=8 \sqrt{2} \times 8 \sqrt{2}=128$

And $N=\frac{1}{2} E$,

Then $N=\frac{1}{2} \times 128=64$

The radius $r=\sqrt{64}=8$

$\boldsymbol{E}_{2}=\mathbf{4 r}$ (The positron energy working against the third wave of dark matter)

And therefore, $E_{2}=\mathbf{2} \times \mathbf{8}=\mathbf{1 6}$

Universal level $-\boldsymbol{n}=9$ : 
Every time the second law of motion is at work, the supportive law of motion is activated:

$E_{\text {support }}=2 E_{\text {applied }}$

Meaning, $\boldsymbol{E}_{\text {support }}=\mathbf{2} \boldsymbol{E}_{2}$

And therefore, $E_{\text {support }}=2 \times 32=64$

\section{Universal level $-n=10$ :}

\section{$E=m_{1} \times m_{2}$}

Then, $P=\mathbf{6 4} \times \mathbf{6 4}=\mathbf{4 0 9 6}$

Here, $\boldsymbol{N}=\boldsymbol{E}$ because nuclear

enhancement has now been able to

balance with $\boldsymbol{M}=\frac{\mathbf{1}}{\mathbf{2}}$ to a ratio of $1: 1$

speed.

Therefore, $\boldsymbol{N}=\mathbf{1} \times \boldsymbol{E}$,

Then $\boldsymbol{N}=\mathbf{4 0 9 6}$

The radius, $r=\sqrt{\mathbf{4 0 9 6}}=\mathbf{6 4}$

$\boldsymbol{E}_{2}=\mathbf{2 r}$ (The positron energy working against the third wave of dark matter)

And therefore, $E_{2}=2 \times 64=128$

\section{$E=m_{1} \times m_{2}$}

Then, $P=\mathbf{3 2} \times \mathbf{3 2}=\mathbf{1 0 2 4}$

Here, $\boldsymbol{N}=\boldsymbol{E}$ because nuclear

enhancement has now been able to

balance with $\boldsymbol{M}=\frac{\mathbf{1}}{\mathbf{2}}$ to a ratio of $1: 1$

speed.

Therefore, $\boldsymbol{N}=\mathbf{1} \times \boldsymbol{E}$,

Then $\boldsymbol{N}=\mathbf{1 0 2 4}$

The radius, $r=\sqrt{\mathbf{1 0 2 4}}=\mathbf{3 2}$

$\boldsymbol{E}_{2}=\mathbf{2 r}$ (The positron energy working against the third wave of dark matter)
And therefore, $E_{2}=2 \times 32=64$
Every time the second law of motion is at work, the supportive law of motion is activated $E_{\text {support }}=2 E_{\text {applied }}$

Meaning, $\boldsymbol{E}_{\text {support }}=\mathbf{2} \boldsymbol{E}_{2}$

And therefore, $E_{\text {support }}=2 \times 16=32$

\section{Universal level $-n=10$ :}

$E=m_{1} \times m_{2}$

Then, $P=\mathbf{3 2} \times \mathbf{3 2}=\mathbf{1 0 2 4}$

Here, $\boldsymbol{N}=\boldsymbol{E}$ because nuclear

enhancement has now been able to

balance with $\boldsymbol{M}=\frac{\mathbf{1}}{\mathbf{2}}$ to a ratio of $1: 1$

speed.

Therefore, $\boldsymbol{N}=\mathbf{1} \times \boldsymbol{E}$,

Then $\boldsymbol{N}=\mathbf{1 0 2 4}$

The radius, $r=\sqrt{\mathbf{1 0 2 4}}=\mathbf{3 2}$

$\boldsymbol{E}_{2}=\mathbf{2 r}$ (The positron energy working

against the third wave of dark matter)

And therefore, $E_{2}=2 \times 32=64$

\section{$E=m_{1} \times m_{2}$}

Then, $P=16 \times 16=256$

Here, $\boldsymbol{N}=\boldsymbol{E}$ because nuclear

enhancement has now been able to

balance with $\boldsymbol{M}=\frac{\mathbf{1}}{\mathbf{2}}$ to a ratio of $1: 1$

speed.

Therefore, $\boldsymbol{N}=\mathbf{1} \times \boldsymbol{E}$,

Then $\boldsymbol{N}=\mathbf{2 5 6}$

The radius, $r=\sqrt{\mathbf{2 5 6}}=\mathbf{1 6}$

$\boldsymbol{E}_{2}=\mathbf{2 r}$ (The positron energy working against the third wave of dark matter)

And therefore, $E_{2}=2 \times 16=32$ 


\begin{tabular}{|c|c|}
\hline Matter & Antimatter \\
\hline $\begin{array}{l}\text { Considering the } 3^{\text {rd }} \text { wave of dark matter that forms the } \\
\text { nucleus: } \\
\text { Therefore } \boldsymbol{N}=\frac{\mathbf{1}}{\mathbf{4}} \boldsymbol{E} \text { this shows that the nucleus of matter } \\
\text { experiences gravity, first as a particle itself and then as an } \\
\text { environment around itself. } \\
\text { And } \boldsymbol{E}=\mathbf{4 N}+\mathbf{4} \text {, this one show that the energy of the } \\
\text { nucleus is already working against the force of gravity on the } \\
\text { level of the } 3^{\text {rd }} \text { wave of dark matter. }\end{array}$ & $\begin{array}{l}\text { Considering the } 2^{\text {nd }} \text { wave of dark matter that forms antimatter: } \\
\text { Therefore } \boldsymbol{N}=\frac{1}{2} \boldsymbol{E} \text { this shows that the nucleus of antimatter } \\
\text { experiences gravity, first as a particle itself and then as an } \\
\text { environment around itself. } \\
\text { And } \boldsymbol{E}=\mathbf{2 N}+\mathbf{2} \text {, this one show that the energy of antimatter } \\
\text { is already working against the force of gravity at the level of } \\
\text { the second wave of dark matter. }\end{array}$ \\
\hline The unified energy field: & The unified energy field: \\
\hline $\begin{array}{l}\text { Since } \boldsymbol{E}=\mathbf{4} \boldsymbol{r}^{2}+\mathbf{4 r} \text {, this is the representation of a complete } \\
\text { energy field forming the nucleus. }\end{array}$ & $\begin{array}{l}\text { Since } \boldsymbol{E}=2 r^{2}+2 r \text {, this is the representation of a complete } \\
\text { energy field forming antimatter. }\end{array}$ \\
\hline $\begin{array}{l}\text { Therefore } E=\mathbf{4}\left(4 r^{2}+4 r\right) \\
\qquad E=16 r^{2}+16 r, \\
\text { This is the unified energy field of the universe, when focused } \\
\text { only on the particles of the universe. }\end{array}$ & $\begin{array}{l}\text { Therefore } E=\mathbf{4}\left(2 r^{2}+2 r\right) \\
\qquad E=8 r^{2}+8 r \\
\text { This is the unified energy field of the universe, when focused } \\
\text { only on the antiparticles of the universe. }\end{array}$ \\
\hline $\begin{array}{l}\text { It was established earlier that, } \mathbf{4} \boldsymbol{r}^{2} \text { represents the neutron and } \\
\text { proton tied in by the strong force and } \mathbf{4 r} \text { represents the electron } \\
\text { driven by the weak force and both } \mathbf{4} \boldsymbol{r}^{2}+\mathbf{4 r} \text { are held together } \\
\text { by the electromagnetic force forming the nucleus. It then } \\
\text { follows that } \boldsymbol{E}=\mathbf{4 N}+\mathbf{4} \text {, is the entire nuclear particle and the } \\
\text { force of dark matter that created it. Under the unified energy } \\
\text { field, the particle is as follows: } \\
\boldsymbol{E}=\mathbf{4}\left(\mathbf{4} \boldsymbol{r}^{\mathbf{2}}+\mathbf{4 r}\right)+\mathbf{4} \\
\text { Then } \boldsymbol{E}=\mathbf{1 6} \boldsymbol{r}^{\mathbf{2}}+\mathbf{1 6 r}+\mathbf{4}\end{array}$ & 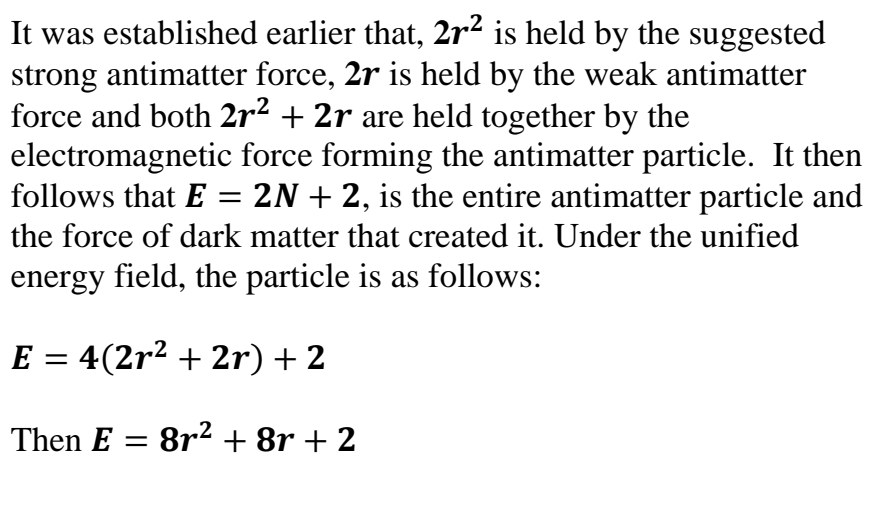 \\
\hline
\end{tabular}

\section{FULFILLED NUCLEAR ENHANCEMENT}

fig 6. Fulfilled nuclei of the environment

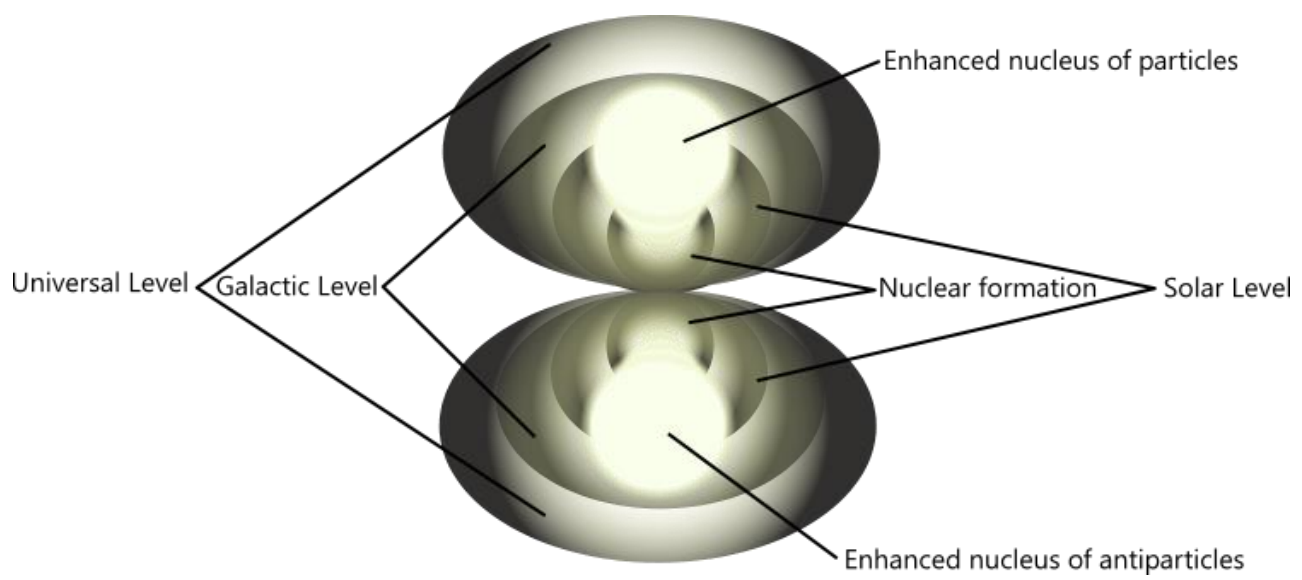

When the nucleus fulfils, there is no need for further enhancement, and this means it has conquered gravity. Therefore, the enhanced nucleus achieves its motionless state, the original state it was as mass with the need to enhance. Only this time the nucleus is fulfilled 
and therefore has no need to enhance. Motionless mass is held together by the root force of mass and therefore, the enhanced nucleus is held together by the root force of mass.

\section{CONCLUSION}

Mass needs to express its potential energy and it does this through physical motion, enhancement and the balance of various forces throughout the physical environment. The energy field of the nucleus unifies so that the force of gravity can be overcome, and this breakthrough is achieved by light particles. The enhancement of the environment from mass through the realm of dark matter and the enhancement of the nucleus is governed by six fundamental laws in the following order:
1. The Subliminal law of motion.
2. Newton's $1^{\text {st }}$ law of motion.
3. Newton's $2^{\text {nd }}$ law of motion.
4. The Supportive law of motion.
5. Newton's $3^{\text {rd }}$ law of motion
6. The Universal law of gravitation.

Dark matter has three waves where mass is moving at different gravitational speed and the first wave (Oclais) is conquered by the unified energy field of the of the universe, the second wave (Diclais) is conquered by the galactic energy field and the third wave (Moclais) is conquered by the solar energy field. Mass transforms itself completely into light that permanently breaks free from the gravity force of dark matter, and therefore it's clear that light conquers every other motion in the environment. When a star goes supernova, it is going through a progressive transformational stage following the process of particle decay. This happens at a point where the star has conquered a certain wave of dark matter/anti-dark matter, thereby moving from the solar level to a galactic level or from the galactic level to the universal level of particle or antiparticles. Over and above the force of gravity, the strong nuclear force, the weak nuclear force and the electromagnetic force, there is a creative and original force of nature that is based at the level of the subliminal law of motion, and this is the root force. Moving from the innermost level of the environment, the forces are layered as follows:

\section{Strong nuclear force. \\ 2. Weak nuclear force. \\ 3. Electromagnetic force. \\ 4. Gravitational force. \\ 5. The root force.}

The gravitational force (realm of dark matter and anti-dark matter) is the force that the nucleus is working hard to conquer, and it is clear that upon conquering this force, particles and antiparticles are kept in balance by the root force of mass. Unlike the force of gravity, the root force is not troubling the nucleus. The theory of enhancement is based on the fact that the environment has a need to fulfil and when the need is fulfilled, it reaches a state of permanent balance.

\section{DATASET}

1. Trevor T Mohlala, Table of Nuclear enhancement, 2021, Figshare HTTPS://FIGSHARE.COM/ARTICLES/DATASET/NUCLEAR_ENHANCEMENT_TABLE/16776124 